Environnement Urbain

Urban Environment

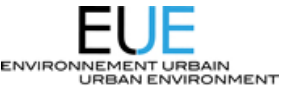

\title{
Crues et inondations majeures des villes de l'Estrie : variations climatiques et modifications anthropiques (Québec, Canada)
}

\section{Diane Saint-Laurent et Marlies Hähni}

Volume 2, 2008

Inondations en milieux urbains et périurbains

Floods in urban and suburban areas

URI : https://id.erudit.org/iderudit/019221ar

DOI : https://doi.org/10.7202/019221ar

Aller au sommaire du numéro

\section{Éditeur(s)}

Institut national de la recherche scientifique

ISSN

1916-4645 (numérique)

Découvrir la revue

\section{Citer cet article}

Saint-Laurent, D. \& Hähni, M. (2008). Crues et inondations majeures des villes de l'Estrie : variations climatiques et modifications anthropiques (Québec, Canada). Environnement Urbain / Urban Environment, 2, 50-72.

https://doi.org/10.7202/019221ar

\section{Résumé de l'article}

Parmi les municipalités du Québec les plus souvent touchées par les inondations, on doit mentionner les municipalités de l'Estrie, qui sont régulièrement affectées par ce phénomène. Les villes de Sherbrooke, Bromptonville, Richmond et Windsor subissent régulièrement les inconvénients et les dommages associés aux inondations. Malgré les efforts consentis par les gestionnaires et responsables municipaux au cours des années, le problème demeure entier et toujours actuel pour ces municipalités riveraines. La présente étude se veut une recension historique des crues et des inondations majeures qui ont marqué les villes de l'Estrie durant la période 1900-2000. Cette recension chronologique est suivie d'une analyse sommaire des conditions hydroclimatiques associées aux événements d'inondation et les principales modifications anthropiques survenues sur ce territoire. 


\title{
CRUES ET INONDATIONS MAJEURES DES VILLES DE L'ESTRIE : VARIATIONS CLIMATIQUES ET MODIFICATIONS ANTHROPIQUES (QUÉBEC, CANADA)
}

\author{
Diane SAINT-LAURENT \\ Marlies HÄHNI
}

\begin{abstract}
I RÉSUMÉ
Parmi les municipalités du Québec les plus souvent touchées par les inondations, on doit mentionner les municipalités de l'Estrie, qui sont régulièrement affectées par ce phénomène. Les villes de Sherbrooke, Bromptonville, Richmond et Windsor subissent régulièrement les inconvénients et les dommages associés aux inondations. Malgré les efforts consentis par les gestionnaires et responsables municipaux au cours des années, le problème demeure entier et toujours actuel pour ces municipalités riveraines. La présente étude se veut une recension historique des crues et des inondations majeures qui ont marqué les villes de l'Estrie durant la période 1900-2000. Cette recension chronologique est suivie d'une analyse sommaire des conditions hydroclimatiques associées aux événements d'inondation et les principales modifications anthropiques survenues sur ce territoire.
\end{abstract}

MOTS-CLÉS - inondation, municipalité, variation hydroclimatique, modification anthropique, Estrie

\begin{abstract}
Among the municipalities of Québec most often hit by floods, those located in the Beauce and Estrie regions, which are regularly affected by this phenomenon, deserve attention. In Estrie, towns such as Sherbrooke, Bromptonville, Richmond and Windsor regularly suffer the consequences and damage associated with flood events. Despite the efforts of local authorities and managers in the past, the problem remains whole and always current for these riverside municipalities. The present study establishes the historical chronology of flood events (high flows and major floods) which had a significant impact on the towns in the Estrie region during the period 1900-2000. A presentation of the flood data is followed by a brief analysis of hydroclimatic conditions associated with flood events and of the major anthropic modifications that have occurred in this area.
\end{abstract}

KEYWORDS - flood, municipality, hydroclimatic variability, anthropic modification, Estrie

\section{REMERCIEMENTS}

Les auteures tiennent à remercier toutes les personnes et organismes qui ont contribué de près ou de loin à la réalisation de cette étude, en particulier le CRSNG, le CRSH (équipe) et le FIR de l'Université du Québec à Trois-Rivières, ainsi que le projet VRM (INRS-UCS) pour leur contribution financière à différents volets de cette étude. Nos remerciements s'adressent aussi à Madame Hélène Liard de la Société d'histoire de Sherbrooke pour sa précieuse aide dans la recherche des documents historiques, le Centre interuniversitaire d'études québécoises (CIEQ-UQTR) pour la consultation des bases de données, ainsi que le ministère de la Sécurité civile du Québec (Directions régionales de la Mauricie-Centre-du-Québec et de Montérégie-Estrie) pour la consultation et la compilation des données ministérielles concernant les inondations. Nos remerciements s'adressent aussi à Mesdames Mariam Ouaaba et Ariane Drouin (UQTR) pour la compilation et la vérification des données climatiques et historiques.

1 Coordonnées des auteurs : Diane Saint-Laurent, géographie, UQTR, C.P. 500, Trois-Rivières, Québec, Canada, G9A 5H7, courriel : diane.saintlaurent@uqtr.ca, Téléphone : (819) 376-50II(3674); télécopieur : (819) 376-5I79 • Marlies Hähni, Sciences de l'Environnement, UQTR, C.P. 500, Trois-Rivières, Québec, Canada, G9A 5H7, courriel : marlies.hahni@uqtr.ca 


\section{INTRODUCTION}

Face aux préoccupations du réchauffement global, on note un nombre croissant d'études traitant des changements climatiques et de leurs impacts sur les différentes composantes biophysiques et sur l'environnement en général. La fréquence des inondations et la diminution des écoulements en rivière font notamment l'objet d'une attention particulière (Douglas et al., 2000 ; Reynard et al., 200 I; Evans et Schreider, 2002; Monirul et al., 2003). Beaucoup de ces travaux s'appuient sur des modèles de simulation climatique basés sur un doublement de $\mathrm{CO}^{2}$, et ce afin de prévoir les impacts des changements globaux sur les régimes hydrologiques à long terme. Les résultats de ces modèles varient beaucoup d'une région à l'autre, indiquant soit une augmentation, soit une diminution des précipitations, affectant à divers degrés le régime d'écoulement des rivières et les risques d'inondation (Panagoulia et Dimou, 1997; Pielke et Downton, 2000 ; Muzik, 200 I ; Evans et Schreider, 2002). II ressort de ces travaux que les effets anticipés des changements climatiques sur les régimes hydrologiques sont difficilement quantifiables en raison des échelles considérées. II importe d'aborder cette problématique à des échelles locales ou régionales pour mieux saisir les incidences climatiques sur les environnements fluviaux. Dans le contexte de ces différents travaux, il paraît intéressant de dresser une recension historique des événements d'inondation depuis un siècle (période 1900-2000), afin de mesurer, d'une part, la fréquence de ces événements à l'échelle régionale et d'autre part, d'identifier les municipalités riveraines de l'Estrie les plus souvent affectées par ce phénomène. On examine aussi les liens avec la séquence des événements d'inondation et les conditions hydroclimatiques (précipitations, débits) enregistrées durant cette période d'un siècle. La recension des événements d'inondation s'appuie sur diverses sources documentaires (archives, rapports ministériels, etc.), alors que les données hydrométéorologiques proviennent de divers registres gouvernementaux (Environnement Canada, CEHQ, etc.). Parallèlement, on examine, pour les villes riveraines de l'Estrie les plus souvent affectées par les inondations, les principales modifications anthropiques survenues durant la période récente. Bien que les facteurs climatiques constituent des facteurs-clés dans la récurrence des événements d'inondation, on doit également considérer les impacts des activités anthropiques comme des facteurs qui influent sur la fréquence et/ou l'aggravation des inondations. Les modifications survenues au cours des dernières décennies (déboisement des rives, occupation des plaines alluviales, etc.) jouent certainement un rôle important quant à l'ampleur et l'intensification des événements d'inondation de cette région.

\section{SOURCE DES DONNÉES ET MÉTHODES D'ANALYSE}

\section{I.I Reconstitution des événements d'inondation (période 1900-2000)}

La reconstitution historique des événements d'inondation pour la région de l'Estrie (Figure I) a été réalisée à partir de données provenant de diverses sources documentaires (rapports gouvernementaux, données ministérielles, monographies, journaux régionaux indexés, etc.). Une première reconstitution des inondations a été compilée essentiellement à partir des fiches d'inondation et autres relevés fournis par le ministère de la Sécurité publique (DRSC du Centre-du-Québec-Mauricie et de la MontérégieEstrie), lesquels couvrent la période postérieure à 1964. Pour ce qui concerne les inondations antérieures à 1964, la compilation a été réalisée à partir de divers documents, dont les rapports de la Commission des Eaux courantes du Québec (période 1912-1952), les rapports ministériels (CECQ, 1944; MRN, 1966; Gagnon et al., 1970, etc.), la revue des différents journaux régionaux indexés par la Société d'histoire de Sherbrooke, la consultation de la base de données du Centre interuniversitaire d'études québécoises, ainsi que des monographies régionales et des articles publiés (Jones, 1999, 2004, etc.). Les journaux régionaux se sont avérés une source importante d'informations pour recenser les événements d'inondation. Ces données indexées sont d'ailleurs souvent exploitées avec d'autres sources documentaires pour reconstituer l'historique des inondations (Shrubsole et al., 1993 ; Jones, 1999; Barriendos et al., 2003). Mentionnons que dans les divers documents consultés, en particulier dans les journaux, les termes «inondations», " débordements » ou "crues » sont parfois utilisés indifféremment et ne sont pas toujours explicites quant à la gravité ou l'ampleur du phénomène. Par exemple, les crues printanières sont parfois désignées comme des inondations majeures, de très fortes crues ou de graves débordements, ce qui peut être confondant quant à la gravité réelle du phénomène observé. Toutefois, plusieurs des documents consultés fournissent généralement des détails précis sur les dégâts causés par les inondations, notamment sur les infrastructures publiques, les biens et les propriétés privés (ponts ou digues endommagés, routes envahies par les eaux, caves inondées, etc.) et le nombre de 
personnes évacuées. Même si certaines informations peuvent être parfois manquantes (ex. niveaux ou débits atteints par la rivière, l'étendue spatiale de l'inondation), il reste que les journaux indexés constituent une source d'informations importante, qui peut être validée avec d'autres sources documentaires, dont les rapports ministériels et les registres climatiques par exemple.

\section{I.2 Données hydrométéorologiques}

Les données météorologiques (précipitations) et hydrologiques (débits) proviennent principalement des divers registres gouvernementaux (Environnement Canada, 2004 et 2002 ; CEHQ, 2000 ; etc.). L'analyse des précipitations moyennes annuelles (PMA) a été réalisée à partir des données climatiques historiques d’Environnement Canada (2004). Les séries analysées (PMA) ont été représentées graphiquement avec une courbe des anomalies climatiques couvrant la période de 1900-2000. La méthode consiste à calculer les écarts entre les valeurs moyennes de la période de référence choisie (d'une durée de \pm 30 ans) et les moyennes annuelles de chacune des années des séries chronologiques sélectionnées. Dans ce cas-ci, la période de référence couvre les années 1971-2000, qui correspondent aux derniers relevés des normales climatiques (Environnement Canada, 200I). À titre d'exemple, la valeur moyenne des précipitations de la station de Sherbrooke (SHE) pour la période de référence 1971-2000 est de l'ordre de I|44,1 mm, alors que la valeur moyenne annuelle de l'année 1925 est de 1351,8 $\mathrm{mm}$, soit un écart de $207,7 \mathrm{~mm}$. La méthode des anomalies climatiques est souvent utilisée pour des analyses régionales comparatives ou pour illustrer des disparités temporelles et spatiales significatives (cf. Jones et Hulme, 1996). Quant aux données hydrologiques, elles proviennent essentiellement des bases de données des stations hydrométriques d'Environnement Canada (2002) et des données informatisées du CEHQ du ministère du Développement durable, de l'Environnement et des Parcs du gouvernement du Québec (CEHQ, 2000). Le tableau I fournit la liste des stations météorologiques et hydrométriques utilisées dans le cadre de cette étude.

\section{RECENSION HISTORIQUE DES INONDATIONS EN ESTRIE (1900-2000)}

\section{I Recension des événements d'inondation à l'échelle séculaire}

Les événements d'inondation en Estrie ont été recensés pour la période de 1900-2000, en tenant compte des fréquences annuelles et mensuelles. La figure 2 représente la fréquence des inondations pour les municipalités les plus souvent affectées par les inondations, soit Bromptonville, Lennoxville, Richmond, Sherbrooke et Windsor (Saint-Laurent et al., 200I), tandis que le tableau 2 fournit la répartition

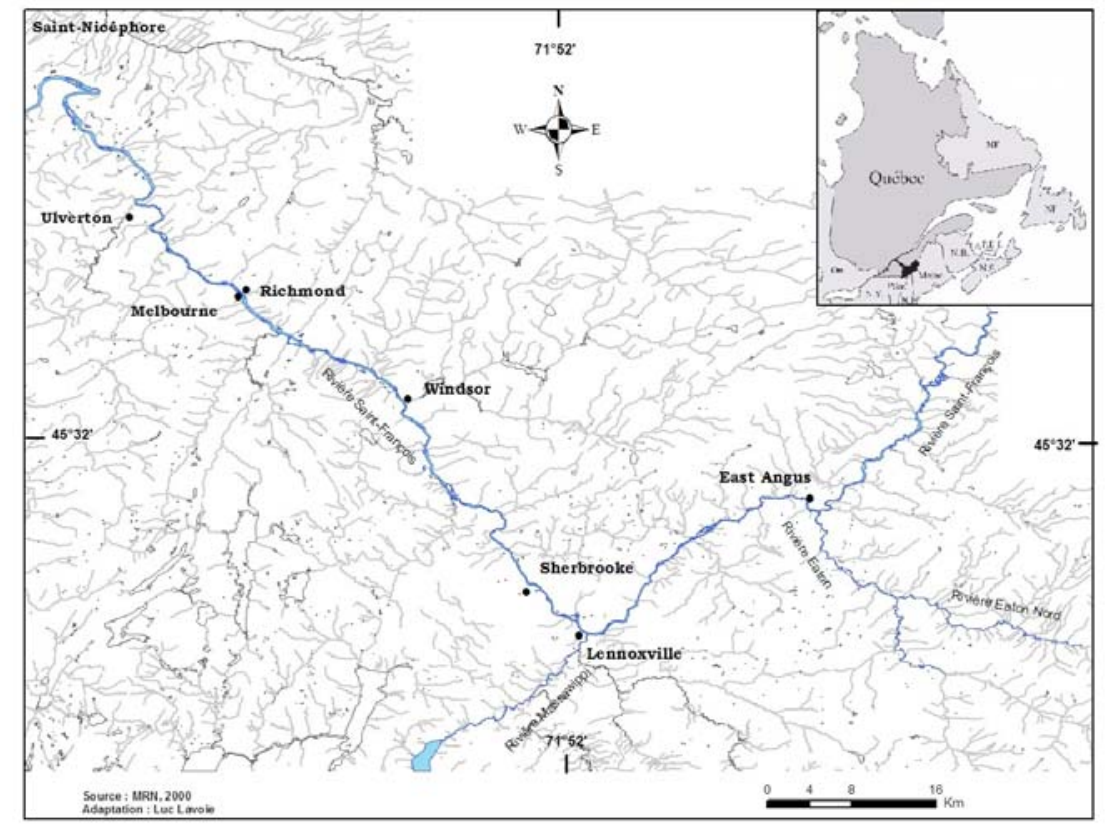

Fig. I - Carte de localisation du bassin de la rivière Saint-François incluant les villes riveraines de l'Estrie 
Tableau I

Stations hydrométéorologiques utilisées pour la compilation et l'analyse des données

\begin{tabular}{|c|c|c|c|c|}
\hline $\begin{array}{l}\text { Stations } \\
\text { météorologiques' }\end{array}$ & Numéro & Localisation & $\begin{array}{c}\text { Altitude } \\
\text { (m) }\end{array}$ & $\begin{array}{c}\text { Période } \\
\text { (précipitation) }\end{array}$ \\
\hline Disraëli (DIS) & 7022000 & $45^{\circ} 55^{\prime} \mathrm{N}-7 \mathrm{I}^{\circ} 24^{\prime} \mathrm{O}$ & 350 & $|908-199|$ \\
\hline Drummondville (DRU) & 7022160 & $45^{\circ} 5^{\prime} 3^{\prime} \mathrm{N}-72 \circ 29^{\prime} \mathrm{O}$ & 82 & $1914-2000$ \\
\hline Lambton (LAM) & 7024000 & $45 \circ 50 ’ \mathrm{~N}-7 \mid \circ 05^{\prime} \mathrm{O}$ & 366 & $1916-1994$ \\
\hline Lennoxville (LEN) & 7024280 & $45^{\circ} 22^{\prime} \mathrm{N}-7 \mid \circ 49^{\prime} \mathrm{O}$ & 181 & $1915-2003$ \\
\hline Sherbrooke (SHE) & 7028124 & $45^{\circ} 26^{\prime} \mathrm{N}-7 \mid \circ 4 I^{\prime} \mathrm{O}$ & 241 & $1904-2000$ \\
\hline $\begin{array}{l}\text { Stations } \\
\text { hydrométriques }^{2}\end{array}$ & Numéro & Localisation & $\begin{array}{l}\text { Superficie du } \\
\text { bassin }\left(\mathrm{km}^{2}\right)\end{array}$ & $\begin{array}{c}\text { Période } \\
\text { (débits enregistrés) }\end{array}$ \\
\hline Rivière Saint-François & 02OE00I & $45^{\circ} 56^{\prime} \mathrm{N}-7|\circ| 6^{\prime} \mathrm{O}$ & 1230 & $1968-1997$ \\
\hline $\begin{array}{l}\text { Rivière Saint-François } \\
\text { Centrale Westbury }\end{array}$ & $02 \mathrm{OE} 007$ & $45^{\circ} 29^{\prime} \mathrm{N}-7 \mid \circ 37^{\prime} \mathrm{O}$ & 3330 & $1929-1987$ \\
\hline Rivière Saint-François & $02 \mathrm{OE} 062$ & $45^{\circ} 39^{\prime} \mathrm{N}-7 \mid \circ 28^{\prime} \mathrm{O}$ & 2930 & $1979-1997$ \\
\hline $\begin{array}{l}\text { Rivière Saint-François } \\
\text { Secteur Richmond }\end{array}$ & 02OF00I & $45^{\circ} 39^{\prime} \mathrm{N}-72 \circ 08{ }^{\prime} \mathrm{O}$ & 9170 & $1915-1965$ \\
\hline $\begin{array}{l}\text { Rivière Saint-François } \\
\text { Secteur Drummondville }\end{array}$ & 02OF002 & $45 \circ 5 \mathrm{I} N \mathrm{~N}-72 \circ 27 ’ \mathrm{O}$ & 9610 & $1925-2000$ \\
\hline $\begin{array}{l}\text { Rivière Saint-François } \\
\text { Secteur Windsor }\end{array}$ & $02 O F 004$ & $45^{\circ} 33^{\prime} \mathrm{N}-72 \circ 00^{\prime} \mathrm{O}$ & 8680 & $1935-1973$ \\
\hline
\end{tabular}

Sources: Environnement Canada 2004, 2002 ; CEHQ, 2000.

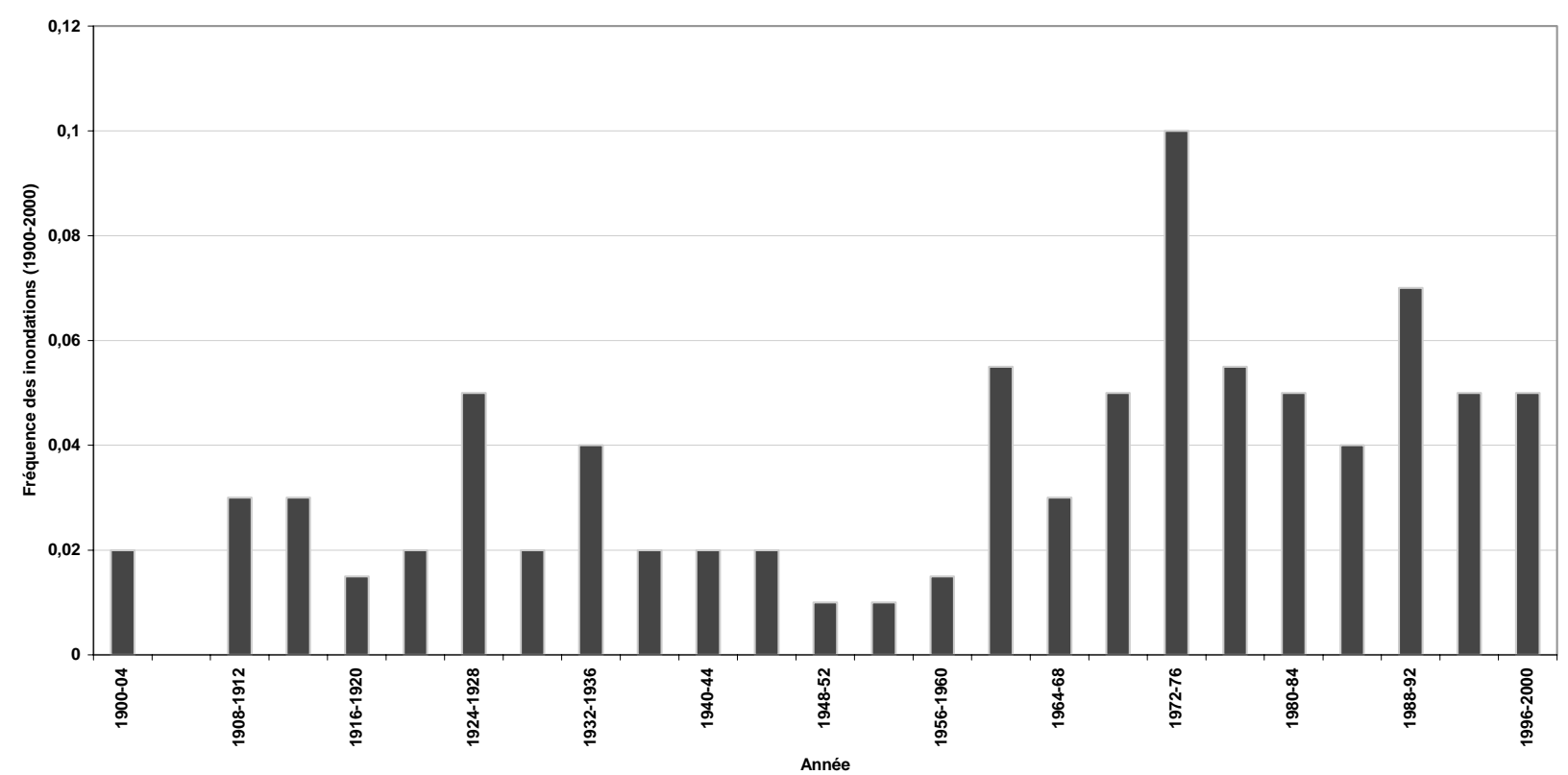

Fig. 2 - Histogramme montrant la fréquence des inondations pour les villes riveraines de l'Estrie 


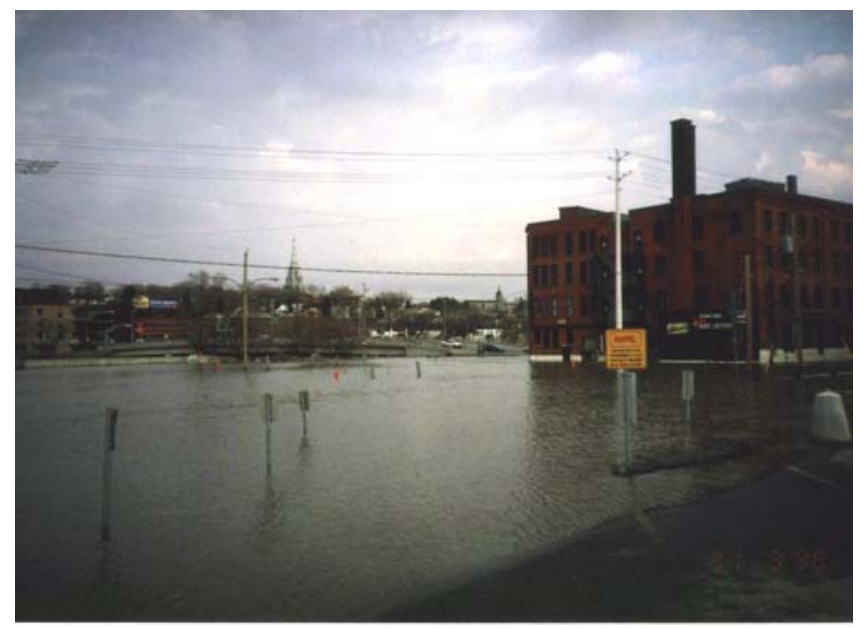

Fig. 3 - Photographie de l'inondation printanière de mars 1998 à Sherbrooke - pont Aylmer (photo de C. Couture)

du nombre d'inondations pour chacune de ces municipalités. Dans certains cas, ces villes ont été soumises à plus d'une inondation annuelle. Ce fut le cas de Sherbrooke et Lennoxville en 1976 ou encore de Sherbrooke et Richmond en 1972. La Ville de Sherbrooke a été particulièrement affectée par les inondations, ce qui s'explique en partie par l'extension importante du domaine urbain dans les zones inondables, mais aussi en raison de la confluence d'importants tributaires (Massawippi, Magog) qui se jettent dans la rivière Saint-François à la hauteur de Sherbrooke. Le débordement de ces tributaires entraîne le rehaussement du niveau de la rivière qui atteint rapidement son débit de «pleins bords » et provoque l'inondation de plusieurs rues du centre-ville de Sherbrooke. La cote critique d'inondation', fournie par le ministère de la Sécurité publique, à la hauteur du Parc Saint-François, face à l'embouchure de la rivière Magog, est de l'ordre de $1273 \mathrm{~m}^{3} / \mathrm{s}$, alors que dans la basse terrasse, en amont, à la hauteur des rues Saint-François Nord et Déziel ${ }^{2}$, le débordement de la rivière peut se produire à un débit de $872 \mathrm{~m}^{3} / \mathrm{s}$. II faut dire qu'une partie des quartiers centraux de Sherbrooke se situe dans la Basse-ville, qui elle-même occupe des niveaux d'élévation relativement bas par rapport à la rivière. En fait, les rues Windsor, Déziel et Saint-François Nord, ainsi que les rues des Grandes Fourches, King Ouest et la rue Wellington Sud sont

I Pour les villes de Bromptonville, Windsor et Richmond, les cotes critiques d'inondation ne sont pas disponibles en mesure de débit. Seuls les niveaux des échelles limnimétriques sont accessibles pour ces municipalités, ce qui demeure peu pratique pour la comparaison débit et récurrence d'inondation.

2 Voir l'article de A. Drouin dans ce même numéro pour la localisation précise des niveaux d'inondation pour ce secteur. périodiquement envahies par les eaux de la rivière Saint-François lors des fortes crues. La figure 3 montre d'ailleurs le débordement de la rivière au cours du printemps de 1998, près de la rue King Ouest, à la hauteur du pont Aylmer. Cette inondation de 1998 et celle de 1982 ont été les pires inondations enregistrées au cours des trente dernières années, et ces inondations ont affecté plusieurs autres municipalités riveraines de l'Estrie. En 1998, le débit maximal enregistré durant cet épisode était de l'ordre de $2050 \mathrm{~m}^{3} / \mathrm{s}$, alors que pour l'inondation de 1982, le débit maximal enregistré était de $2420 \mathrm{~m}^{3} / \mathrm{s}$ à la station de référence (station 02OF002).

Enfin, en examinant la fréquence des inondations du bassin de la rivière Saint-François à l'échelle du siècle (1900-2000), on note deux périodes marquées par une augmentation des inondations, soit une première période entre 1924 et 1940 , et une seconde période entre 1970 et 1998 (Figure 2). Par contre, la période entre 1950-1960 se caractérise plutôt par une faible occurrence des inondations, qui se traduit également par une baisse pluviométrique durant ces années (voir section 4.I). À titre complémentaire, l'annexe fournit en détails les dates des événements d'inondations recensées pour la période 1925-2000 pour les principales municipalités de l'Estrie, ainsi que les débits enregistrés à la station de référence 02OF002, qui constitue la série hydrométrique la plus complète pour ce secteur. Pour ce qui a trait aux caractéristiques saisonnières des inondations, on note une forte disparité dans la fréquence de ces événements, qui se produisent le plus souvent durant les mois de mars et avril (Figure 4). Durant cette période printanière, les inondations sont généralement conditionnées par la fonte rapide du couvert nival, 
combinées le plus souvent à des pluies abondantes et aux embâcles, ce qui est d'ailleurs le trait dominant des bassins hydrographiques du centre du Québec (Gagnon et al., 1970; Llamas et Triboulet, 1979). D'ailleurs, l'étude hydrologique réalisée par Llamas et Triboulet (1979), dans le bassin moyen du SaintLaurent, pour la période 1937-1971, indique en effet que les pointes de crue se produisent en mars et avril, et plus fréquemment entre le 10 et 15 avril. L'analyse réalisée par Gagnon et al. (1970) sur les crues exceptionnelles des rivières Saint-François et Chaudière pour une période antérieure, soit 1912 et 1964, conduit à des conclusions semblables. Ces derniers auteurs soulignent par ailleurs que la fonte rapide du couvert neigeux, accompagnée de pluies abondantes, constituent les conditions les plus propices aux inondations printanières.

En examinant la récurrence des inondations sur la figure 4 pour la période 1900-2000, on constate que les dernières décennies sont davantage marquées par des crues printanières qui se produisent plus souvent en mars qu'en avril, ce qui pourrait traduire un changement du régime hydrologique lié à des conditions printanières plus précoces. On note par ailleurs une recrudescence des inondations pour les mois de décembre, janvier et plus particulièrement le mois de février. Cela révèle aussi un changement de saisonnalité dans la fréquence des événements d'inondation, ce qui pourrait être associé à un adoucissement des températures depuis les dernières décennies. Les températures hivernales des mois de décembre, janvier et février sur près d'un siècle (Figure 5) montrent en effet une légère hausse, en particulier pour le mois de février. Toutefois, cette tendance est beaucoup moins marquée pour les mois de décembre et janvier. Enfin, les inondations du mois d'août ont été beaucoup plus fréquentes depuis le milieu du $X X^{e}$ siècle. L'analyse détaillée des

Tableau 2

Recension du nombre d'inondations par municipalité en Estrie (période 1900-2000)

\begin{tabular}{lc}
\hline Municipalités riveraines & Nombre d'inondations* \\
\hline Bromptonville & 31 \\
\hline Lennoxville & 28 \\
\hline Richmond & 39 \\
\hline Sherbrooke & 53 \\
\hline Windsor & 22 \\
\hline *Les inondations dont la date et l'année sont incertaines ont été exclues du tableau.
\end{tabular}

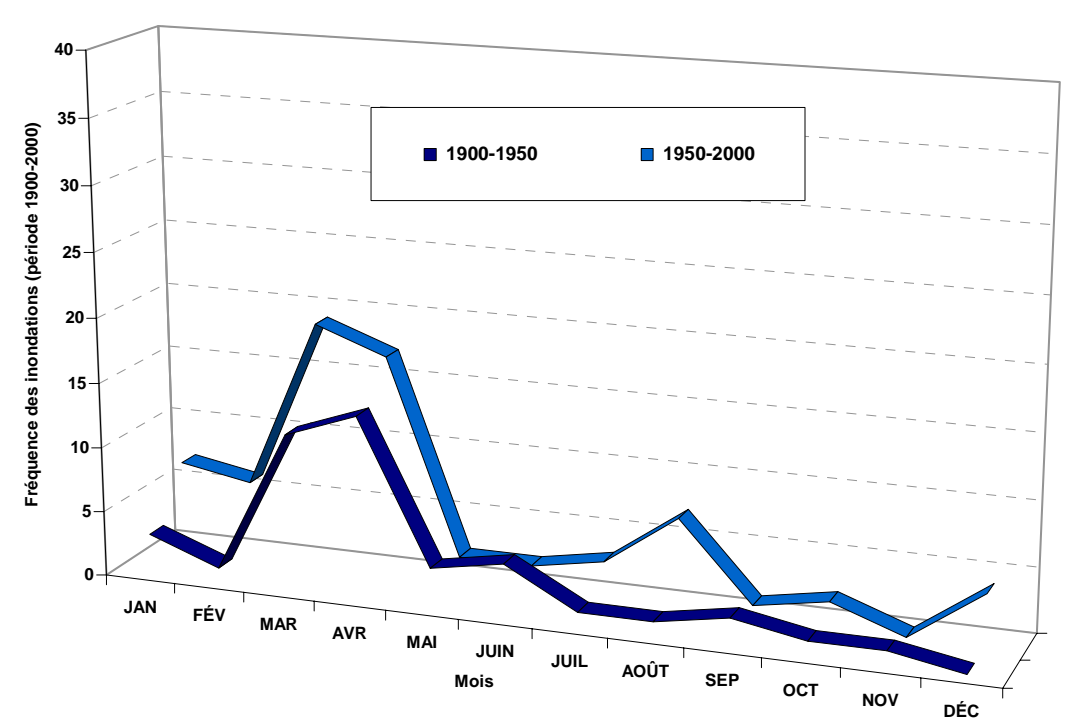

Fig. 4 - Fréquence saisonnière des inondations des municipalités riveraines de l'Estrie 
précipitations mensuelles du mois d'août (moyennes mobiles) à différentes stations (DIS, DRU et SHE) indique en effet une légère tendance à l'augmentation des épisodes pluviométriques durant la seconde moitié de ce dernier siècle pour cette période de l'année (Figure 6). Cette tendance à des épisodes pluviométriques plus fréquents en fin d'été et au début de l'automne semble d'ailleurs se manifester dans toute la région des Bois-Francs et de l'Estrie selon l'étude de Siew-Yan-Yu et al. (1998).

\subsection{Recensement des inondations majeures}

En se basant sur les rapports de la Commission des eaux courantes du Québec, les données hydrologiques et d'autres sources documentaires (rapports ministériels, journaux régionaux indexés, etc.), il a été possible d'inventorier les plus fortes inondations du bassin de la rivière Saint-François pour les municipalités riveraines de l'Estrie. Les inondations de 1924, 1927, 1942, 1943, 1976, 1982, 1994, 1996 et 1998 figurent parmi les plus importantes inondations de cette région (Tableau 3). Les inondations de 1942 , 1943 et 1982 sont considérées comme les pires inondations du $X X^{e}$ siècle, ayant affecté plusieurs municipalités de la région et ayant entraîné d'importants dommages matériels (bris de digues, ponts déchaussés, caves inondées, etc.). Ces inondations qualifiées de «majeures» ou « catastrophiques » sont le plus souvent associées à des pertes matérielles importantes qui ont affecté plusieurs municipalités à la fois. L'inondation du printemps de 1982 est souvent citée comme l'inondation la plus désastreuse des dernières décennies, ayant entraîné le débordement de plusieurs rivières et des dommages matériels considérables.
Cette inondation de 1982 a d'ailleurs fait l'objet d'une analyse détaillée par Lamb (1982) et Hoang (1982). Ces derniers expliquent que ce sont des pluies exceptionnelles de plusieurs heures, provoquées par le passage de deux fronts successifs, qui sont à l'origine de cet événement catastrophique. Ces pluies diluviennes ont entraîné la fonte rapide du couvert nival, anormalement épais pour cette période de la miavril, soit un couvert nival ayant des valeurs de 50 à $200 \mathrm{~mm}$ au-dessus de la médiane (Lamb, 1982). La plus forte valeur enregistrée était de l'ordre $67,2 \mathrm{~mm}$ de pluies tombées en moins de 24 heures dans le bassin amont de la Saint-François (Val Racine). On estime que de telles pluies exceptionnelles correspondent à des périodes de retour comprises entre 125 et 150 ans (Hoang, 1982 ; Lamb, 1982). Outre l'inondation de 1982, on compte aussi plusieurs autres inondations printanières majeures qui ont affecté les villes de l'Estrie, dont les inondations de 1992, 1994 et 1998. Les inondations de 1994 et de 1998 ont été particulièrement sévères pour la Ville de Sherbrooke. Enfin, les inondations des étés de 1942 et de 1943 sont elles aussi souvent citées parmi les pires du siècle dernier. L'inondation de 1942 s'apparente d'ailleurs en gravité à celle de 1982, causant le débordement de plusieurs rivières en Estrie et affectant de nombreuses municipalités riveraines. Pour les inondations des étés de 1942 et 1943, ce sont des pluies abondantes de plusieurs jours qui auraient été la cause principale de ces inondations majeures. On a enregistré des valeurs de 257, $2 \mathrm{~mm}$ de pluies entre le 14 et 16 juin 1942 et de $303,4 \mathrm{~mm}$ entre le 15 et 17 juin 1943, alors que la moyenne mensuelle durant cette période de l'année est de l'ordre de $120,1 \mathrm{~mm}$ de pluies suivant les données de la station de Sherbrooke pour la période 1902-1994 (Environnement Canada, 2004). Selon les rapports de Boucher et al. (1952, 1953), les pluies torrentielles de juin 1942 et 1943, causées par des.

Tableau 3

Inondations majeures recensées dans les villes de l'Estrie

\begin{tabular}{|c|c|c|c|c|c|c|}
\hline Année & Inondation majeure & Bromptonville & Lennoxville & Richmond & Sherbrooke & Windsor \\
\hline 1998 & 29 mars au 4 avril & $x$ & $x$ & $x$ & $x$ & $x$ \\
\hline 1996 & 17 au 25 janvier & $x$ & - & - & $x$ & $x$ \\
\hline 1994 & 7 au 18 avril & - & $x$ & $x$ & $x$ & - \\
\hline 1982 & I7 au I9 avril & $x$ & $x$ & $x$ & $x$ & $x$ \\
\hline 1976 & 26 mars au 3 avril & - & $x$ & - & $x$ & $x$ \\
\hline 1943 & I6 au I7 juin & $x$ & $x$ & $x$ & $x$ & - \\
\hline 1942 & 14 au 15 juin & $x$ & $x$ & $x$ & $x$ & $x$ \\
\hline 1936 & 13 au 19 mars & $x$ & - & $x$ & $x$ & - \\
\hline 1928 & 7 au 9 avril & $x$ & $x$ & $x$ & $x$ & $x$ \\
\hline 1927 & 3 au 5 novembre & - & - & $x$ & $x$ & - \\
\hline 1924 & 8 au II septembre & $x$ & $x$ & $x$ & $x$ & $x$ \\
\hline
\end{tabular}

Note : le signe ( -) signifie qu'aucune inondation a été signalée pour la date inscrite. 


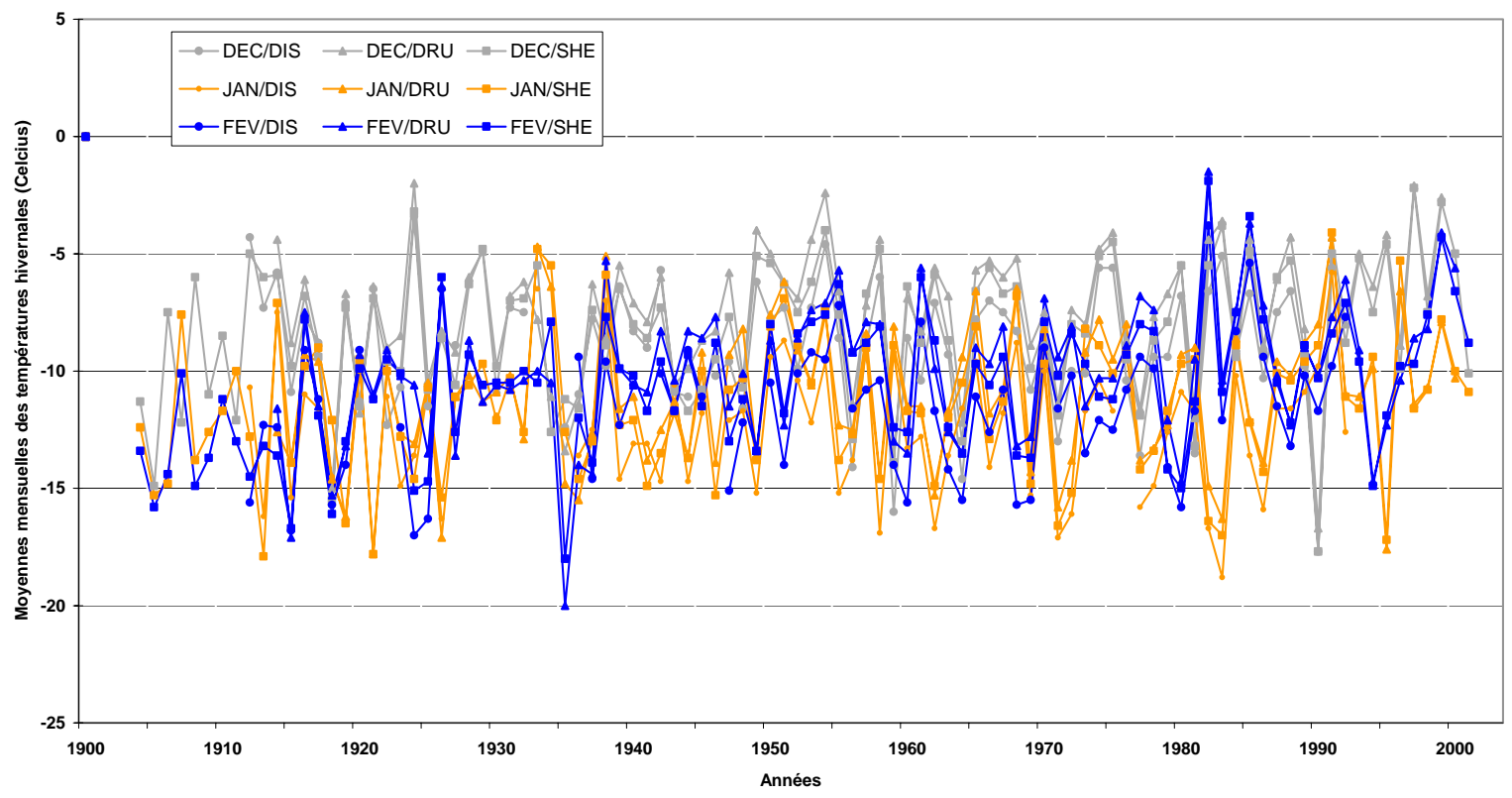

Fig. 5 - Moyennes mensuelles des températures hivernales des stations DIS, DRU et SHE

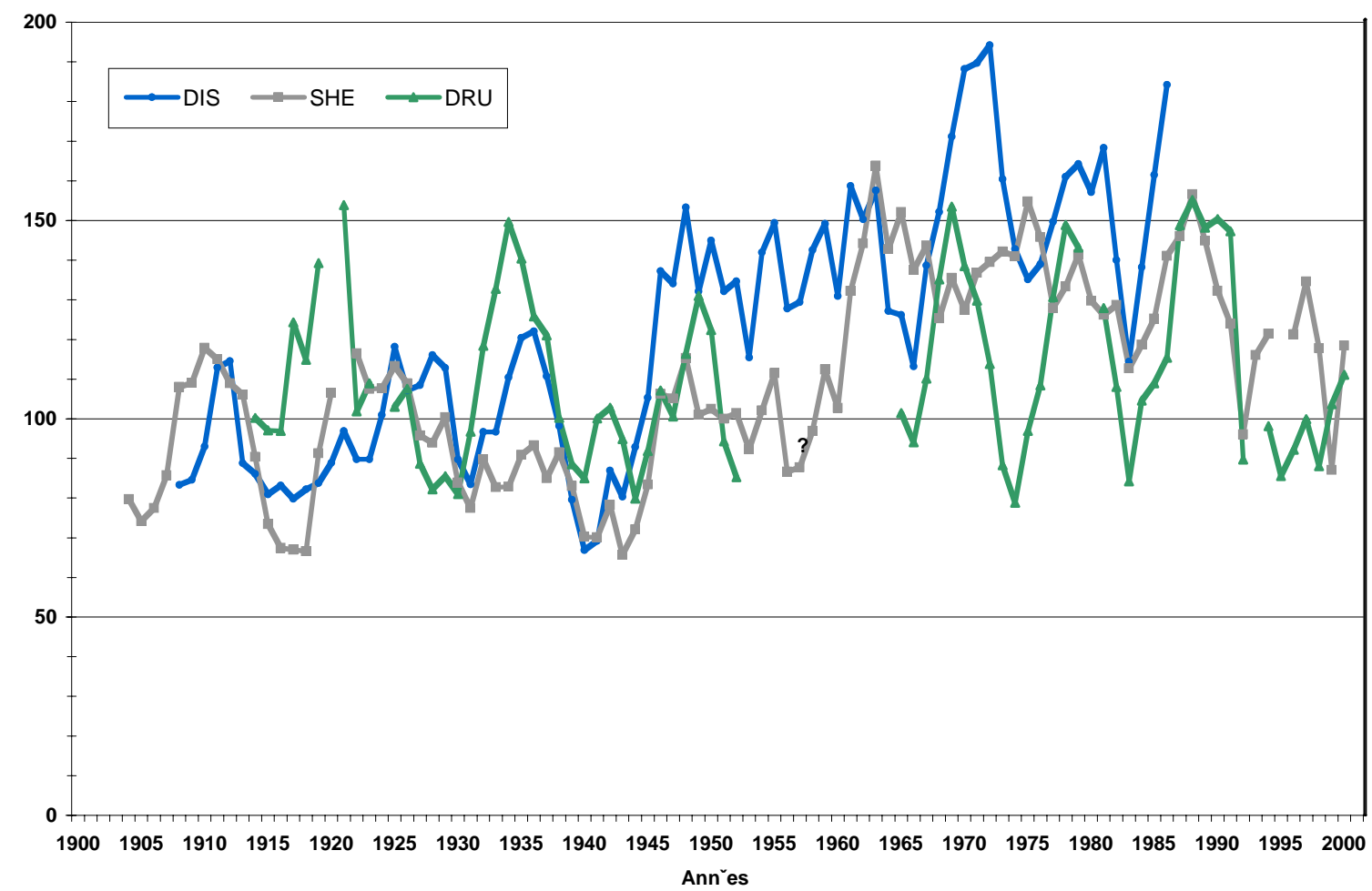

Fig. 6 - Moyennes mobiles des précipitations mensuelles du mois d'août 
cellules orageuses, auraient fait monter rapidement le niveau de plusieurs rivières du bassin de la SaintFrançois (Magog, Au Saumon, Eaton, etc.), et ce trop plein d'eau soudain a généré de graves inondations pour plusieurs municipalités riveraines, dont la Ville de Sherbrooke

\section{VARIABILITÉ HYDROCLIMATIQUE}

\section{I Variabilité interannuelle des précipitations}

L'analyse des précipitations constitue un facteur clé pour comprendre la dynamique des écoulements en rivière et la fréquence des inondations assujettie aux conditions du régime hydrologique. Pour cette analyse, nous avons considéré les précipitations moyennes annuelles (PMA) des données des stations de Disraëli (DIS), Drummondville (DRU), Lambton (LAM), Lennoxville (LEN) et Sherbrooke (SHE). Ces données regroupent les précipitations totales (neiges et pluies) qui sont représentées suivant la compilation des moyennes annuelles fournie par Environnement Canada. De ces précipitations totales, le tiers environ tombe sous forme de neige. La figure 7 fournit d'ailleurs la distribution des précipitations (neiges et pluies) sur près d'un siècle à Sherbrooke. On remarquera que les années 1933, 1962, 1971, 1972 et 1976 sont des années où les accumulations en neige furent parmi les plus importantes, et certaines d'entre elles (1933, 1962 et 1976) coïncident avec des inondations printanières dont les débits enregistrés étaient relativement élevés, en particulier pour les années 1933 et 1976 (Annexe). En examinant la distribution des précipitations (PMA) de la série chronologique, on note une importante variabilité interannuelle pour l'ensemble des stations (Figure 8). Le graphique montre soit des périodes caractérisées par des pics pluviométriques, notamment au début du dernier siècle, entre 1917-1922 et 1936-1946, et plus tard entre 1970-1990, et inversement, des déficits pluviométriques centrés principalement sur les années 1950-1960. En examinant plus attentivement les anomalies des précipitations des stations de DRU et SHE (Figures 9 et 10), on note que les années qualifiées de «pluvieuses 》 ont été 1907, 1912, 1918, 1922, 1936, 1942, 1954, 1974, 1976, 1983 et 1990, tandis que les années déficitaires ont été 1914, 1915, 1926, 1930, 194I et 1956 principalement. Bien que les distributions présentées soient relativement différentes d'une série à l'autre, on peut néanmoins déceler une tendance généralisée à la hausse des précipitations, en particulier pour la période 19701990. Au-delà de cette période, une tendance à la baisse est notée pour les stations de DRU et SHE.

\subsection{Variabilité du régime hydrologique}

Les valeurs enregistrées dans les différentes stations hydrométriques de la rivière Saint-François, situées à proximité des villes de Richmond, Sherbrooke, Windsor et Drummondville, indiquent généralement une forte variabilité des écoulements journaliers (moyens et maximums), ce qui est facilement repérable sur les séries hydrologiques de longue durée en général. On note que la période entre 1970 et 1998 se caractérise par une hausse marquée des écoulements en rivière, qui s'observe dans la plupart des stations analysées (Figure II). Les années 1982, 1994, 1996 et 1998 constituent, par ailleurs, les années au cours desquelles les débits les plus élevés ont été enregistrés, et les années 1927, 1928, 1936, 1939 et 1942 représentent également des années de forts débits (Annexe). En somme, en examinant la distribution des écoulements (débits moyens et maximums) sur près d'un siècle, on peut reconnaître une période de débits plus élevés au début du dernier siècle, qui s'étend entre les années 1927 et 1943, et une deuxième période plus marquée en terme d'hydraulicité, entre les années 1970 et 1998. En revanche, la période entre 1964 et 1968 est plutôt caractérisée par une diminution des écoulements. On peut dire en fait que les années 1928, 1936, 1976, 1982, 1994, 1996 et 1998 ressortent comme des années de forts débits dans la majorité des stations analysées.

En comparant les séries de données de précipitations avec celles des débits analysés, on note rapidement que les années de forte pluviosité sont généralement associées aux années marquées par des débits plus élevés (Figures 9 à $\mathrm{II}$ ). La comparaison entre les années d'inondations majeures et les valeurs des débits maximums révèle également que la majorité des dates d'occurrence de ces événements correspondent à celles des débits maximaux. C'est le cas notamment des années 1924, 1927, 1939, 1942, 1943, 1976, 1982, 1994 et 1998. Outre ces années, la relation entre les débits annuels maximaux enregistrés et l'occurrence des inondations majeures recensées dans les rapports consultés apparaît de façon évidente.

\subsection{Relation entre les épisodes pluviométriques et hydrologiques et les inondations}

En comparant les séries de données de précipitations (PMA) avec celles des débits enregistrés (DAM), on note que les années de forte pluviosité sont généralement associées aux années marquées par des débits plus élevés. Cette relation apparaît 


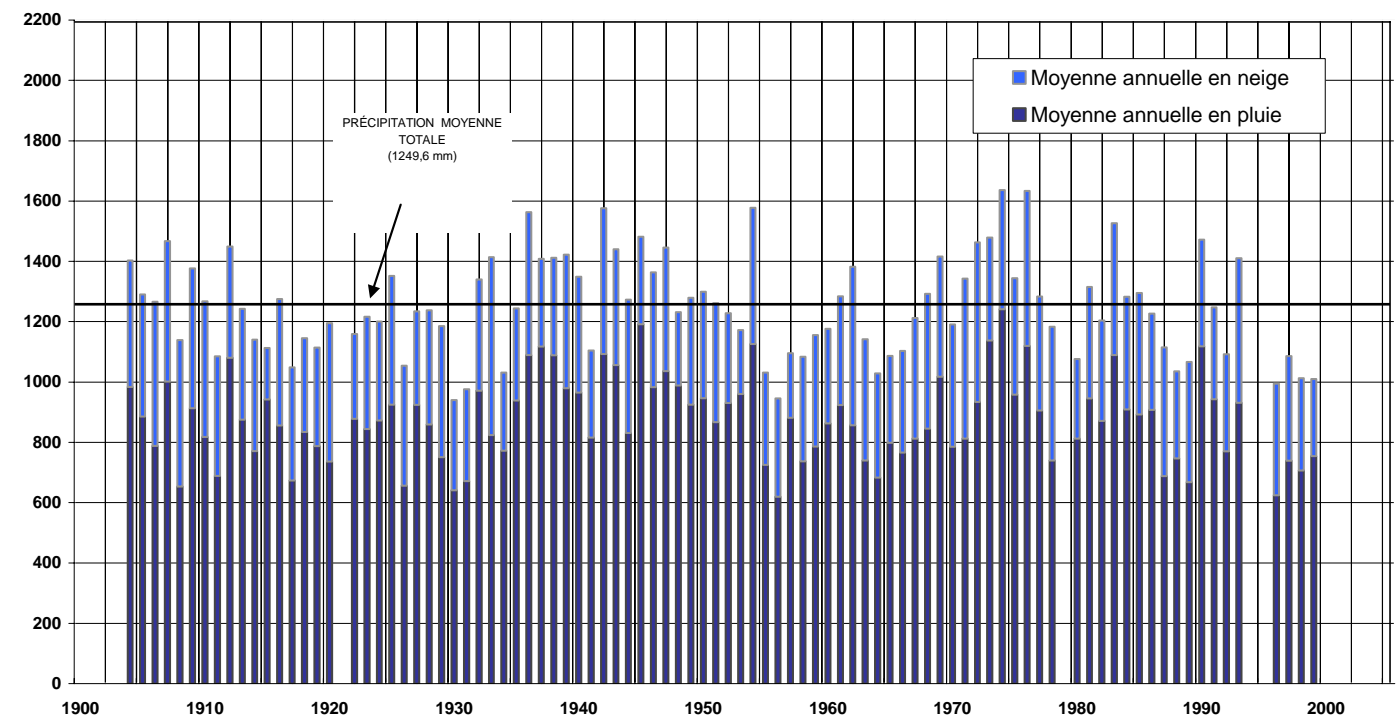

Fig. 7 - Moyennes annuelles des précipitations en pluie et en neige à Sherbrooke

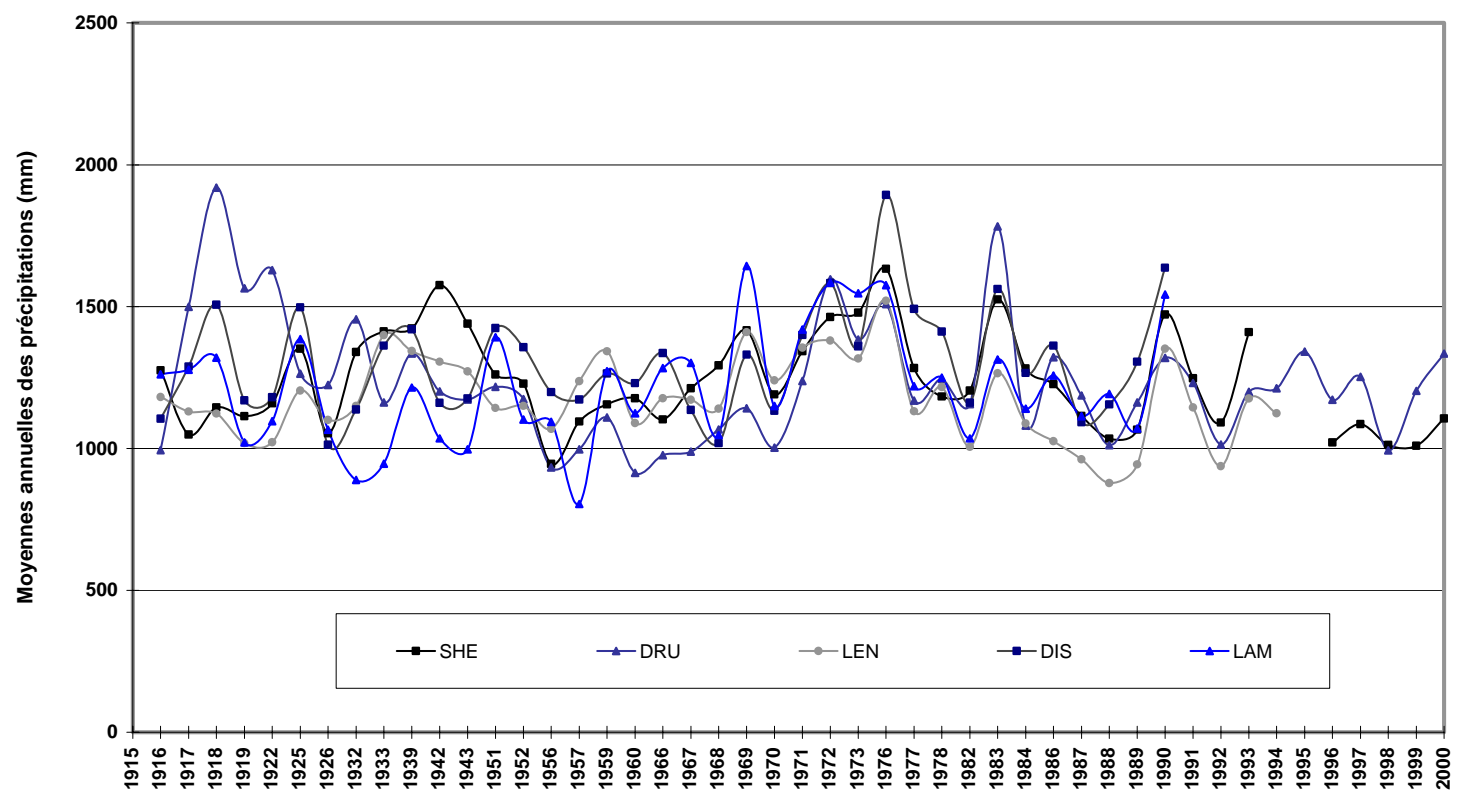

Fig. 8 - Moyennes annuelles des précipitations totales des stations DIS, DRU, LAM, LEN et SHE 
EUE • Crues et inondations majeures des villes de l'Estrie • a-60

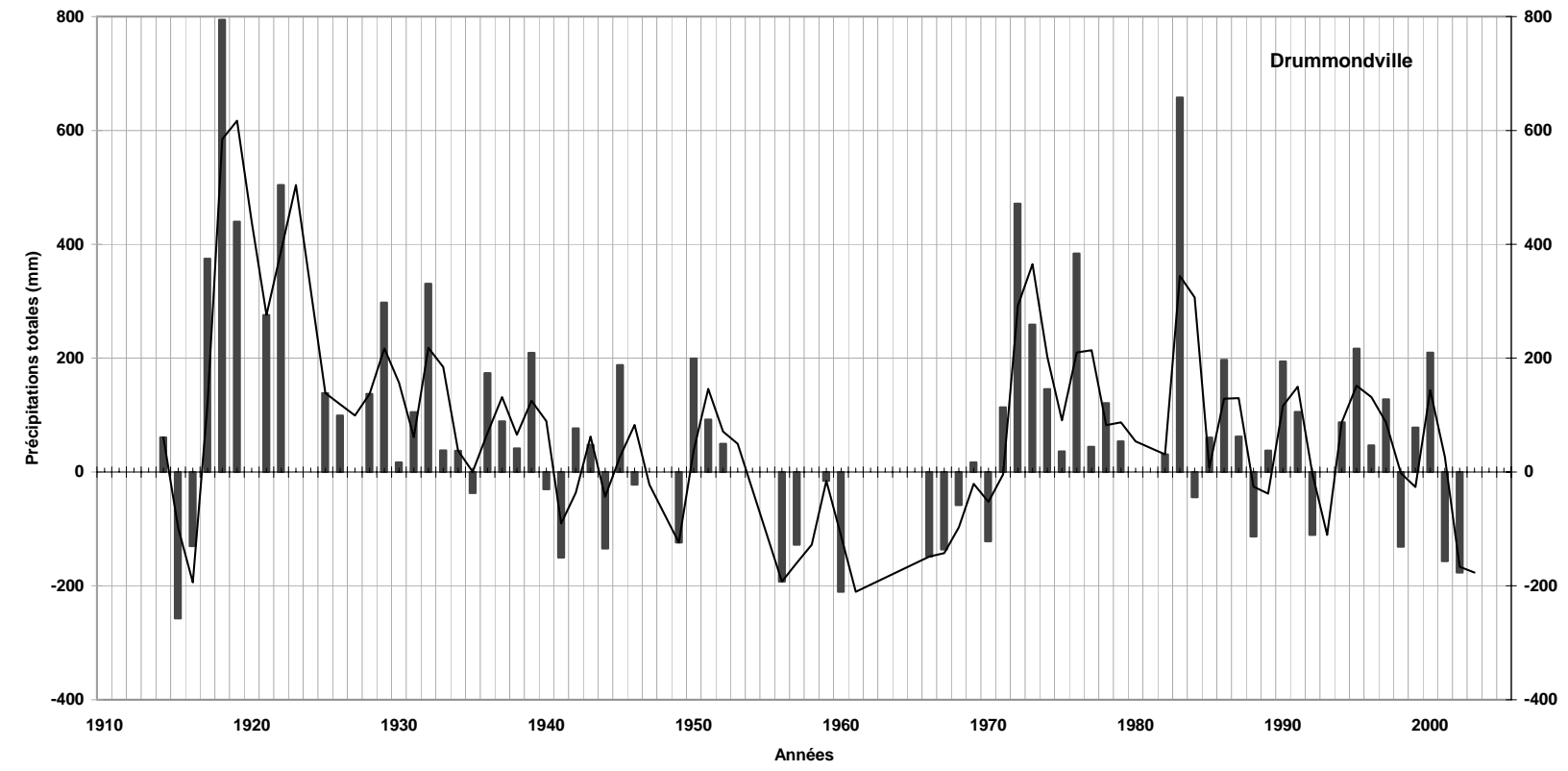

Fig. 9 - Diagramme des anomalies des moyennes annuelles des précipitations à Drummondville

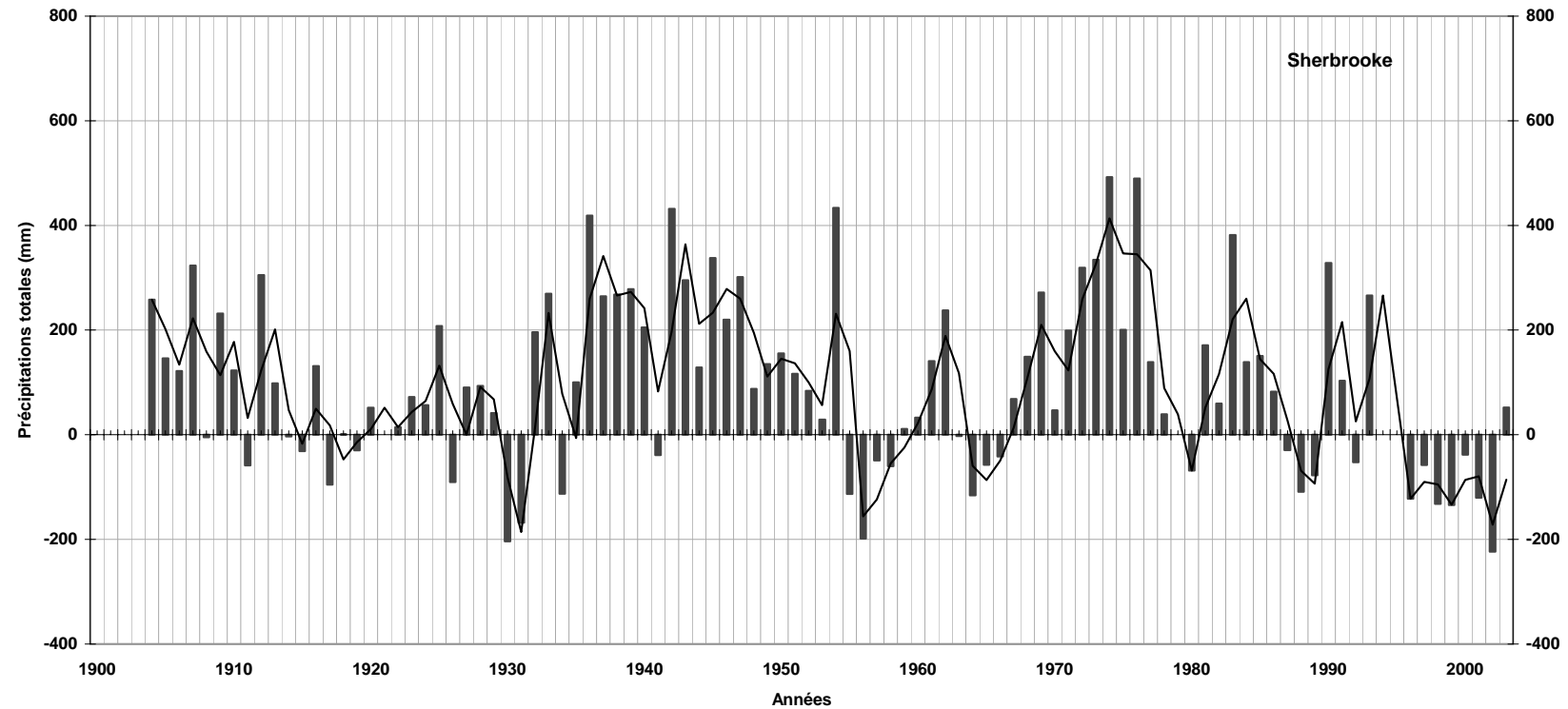

Fig. 10 - Diagramme des anomalies des moyennes annuelles des précipitations à Sherbrooke 


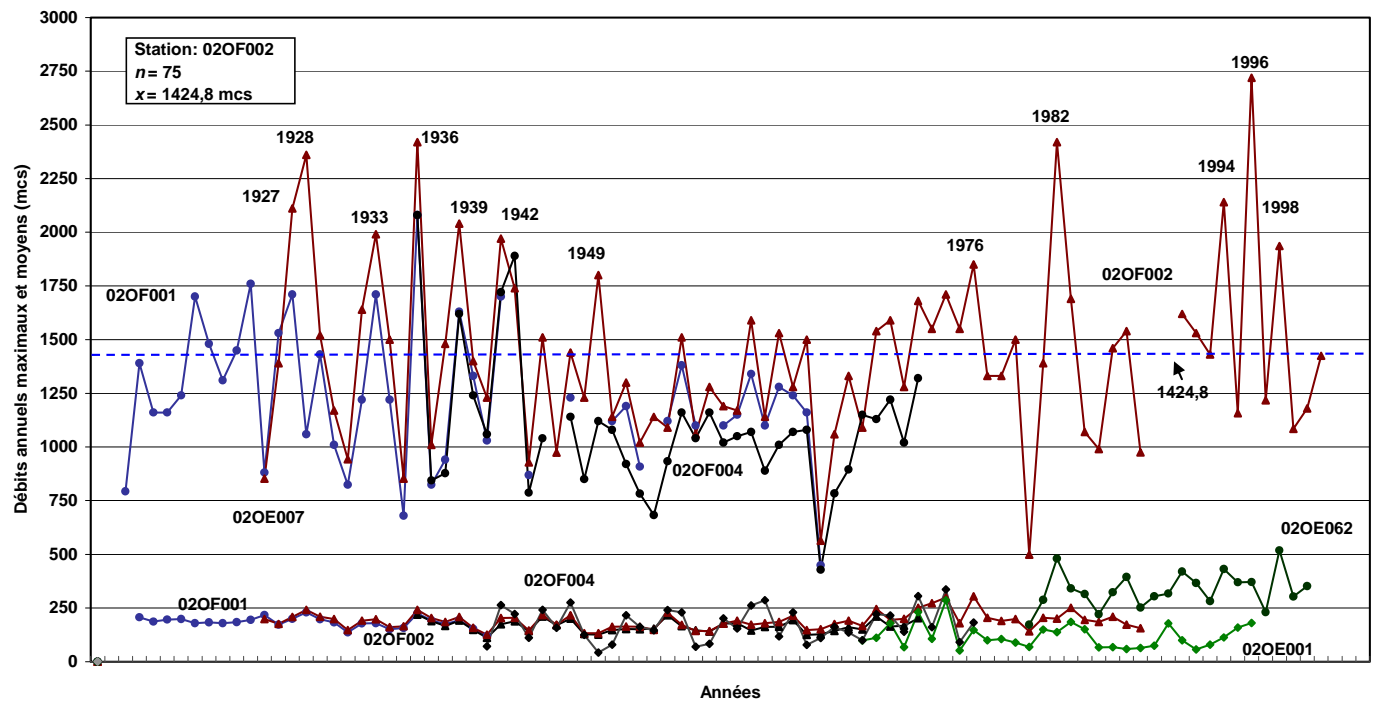

Fig. I I - Moyennes annuelles et maximales des débits à différentes stations hydrométriques

toutefois beaucoup plus nette entre ces deux séries de variables (PMA et DAM) pour les stations météorologique et hydrométrique de Drummondville (Figures 9 et II), toutes deux situées à proximité l'une de l'autre. Par ailleurs, la comparaison entre les années identifiées à des débits journaliers maximaux et les inondations majeures révèle que la majorité des dates d'occurrence de ces épisodes correspondent à celles des débits extrêmes, notamment pour les années 1927, 1928, 1936, 1939, 1942, 1976, 1982, 1994, 1996 et 1998 (Annexe). Toutefois, l'inondation de l'été 1943, qui a été recensée comme une inondation majeure dans les registres, aurait atteint un seuil de débit maximal de $1740 \mathrm{~m}^{3} / \mathrm{s}$, soit légèrement moins élevé que les autres années identifiées comme des années marquées par des inondations majeures. II reste que le seuil atteint lors de cette inondation se situe au $13^{\mathrm{e}}$ rang des 56 autres débits maximaux enregistrés entre 1925-2000 (voir Annexe), ce qui est loin d'être négligeable. Pour ce qui est des années 1901, 1913, 1915 et 1924, qui sont aussi des années identifiées à des inondations importantes dans les rapports gouvernementaux (CECQ, 1952), l'absence de registres hydrométriques rend toute comparaison impossible. Enfin, la relation entre les débits annuels maximaux enregistrés et l'occurrence des inondations majeures recensées dans les rapports consultés apparaît évidente, surtout si l'on compare l'ensemble des séries. On note en effet une distribution comparable entre les séries des données pluviométriques, hydrologiques et les inondations pour la période 1900-2000. Enfin, les débits maximaux les plus élevés se concentrent surtout durant la période de 1970 à 2000, qui coïncide avec des inondations majeures, dont celles de 1982, 1994, 1996 et 1998.

Enfin, en comparant le tableau des inondations recensées et les débits annuels maximaux de la station 02 OF002 (Annexe), on constate que certaines dates d'inondation présentent un débit relativement faible, notamment pour les années 1930, 1931, 1935, 1954, 1962, 1963, 1979, 1980 et 1985. En examinant les documents d'archives pour ces années, on voit que ces dates réfèrent à des inondations plus ou moins mineures. Certaines inondations sont davantage associées à des tributaires de la Saint-François, notamment le long des rivières Coaticook et Massawippi, ce qui explique que les débits enregistrés à la hauteur de la centrale de Drummondville ne permettent pas de détecter tous les événements d'inondation, notamment ceux situés dans des secteurs en amont de la centrale. II faut souligner aussi que certaines inondations sont très localisées, par exemple, celles causées par la formation d'embâcles, qui provoquent des débordements dans des secteurs spécifiques. En raison de leur localisation et de leur étendue, ces embâcles ne sont pas toujours décelés dans les registres hydrologiques, d'où l'importance de combiner différentes sources de données. À titre d'exemple, les années d'inondation de 1930, 1935, 1963 et 1979, causées par des embâcles, ne sont pas identifiées à partir des données de débits maximaux 
enregistrées en aval (station 02OF002). Dans ces cas précis, l'information provenant des documents d'archives vient en quelque sorte compléter les données des registres hydrométriques, assurant ainsi une meilleure couverture de la recension des inondations, même pour des crues mineures. Enfin, les années qui couvrent la période entre 1950 et 1958 ne réfèrent à aucune mention d'inondation dans les sources consultées, sauf l'année 1957, où l'on mentionne que les eaux de la Saint-François ont été très élevées, mais insuffisamment pour entraîner le débordement de la rivière.

En regard à l'augmentation de la fréquence des inondations dans le bassin de la rivière Saint-François depuis les années 1970, et plus particulièrement entre les années 1970 et 1990, on note une certaine correspondance avec l'augmentation des inondations recensées à l'échelle canadienne sur près d'un siècle. Les travaux de Tucker (2000) indiquent en effet une hausse du nombre des inondations dès le début du dernier siècle (1910-1920), mais surtout après les années 1970. Tucker (2000) propose des causes multiples à cette hausse des inondations qu'il résume ainsi: « ... A number of factors may explain this trend: the occurrence of more extreme events, an increase in disasters proportional to the increase in population, better reporting of events in more recent years and/or increased concentration of population in vulnerable areas » (Tucker, 2000 , p. 78). Tous ces facteurs peuvent en effet intervenir de façon concomitante sur l'augmentation et/ou l'aggravation des phénomènes d'inondation, et l'intérêt premier est de tenter de reconnaître la prédominance d'un facteur sur un autre dans le déroulement de ces événements. En ce qui concerne plus spécifiquement les inondations du bassin de la rivière Saint-François, il apparaît que les facteurs climatiques, notamment les épisodes pluviométriques marqués et la fonte rapide du couvent nival, génèrent les inondations les plus importantes de cette région (Gagnon et al., 1970). On doit considérer également les impacts des activités anthropiques sur la fréquence ou l'ampleur des phénomènes d'inondation. Les modifications survenues (déboisement intensif, occupation progressive des rives, etc.) au cours du dernier siècle, par exemple, ont pu avoir une incidence marquée sur l'intensité ou la gravité de ce phénomène. Sans être en mesure de répondre adéquatement aux impacts réels des facteurs anthropiques sur l'aggravation des inondations, on peut néanmoins penser que le développement urbain actuel, notamment dans les villes moyennes ou de fortes densités, comme Sherbrooke ou Drummondville, qui connaissent une importante croissance de leurs zones urbaines, peuvent avoir une incidence marquée sur ce phénomène. L'extension du domaine urbain, notamment en périphérie des centres urbains, et même dans les zones riveraines et le long des plaines inondables, peut être mis en parallèle avec la croissance démographique qu'ont connue ces villes au cours des dernières décennies (Tableau 4). À titre indicatif, la Ville de Sherbrooke a connu une assez forte croissance de sa population après les années quarante, liée à sa vitalité économique, ce qui a entraîné nécessairement un développement urbain important tout autour des quartiers centraux et périphériques et le long de la rivière Saint-François, souvent au détriment d'anciennes zones agricoles ou même de terres boisées. L'augmentation progressive des surfaces urbanisées, aux dépens de ces surfaces « naturelles ou semi-naturelles », entraîne par la force des choses des modifications marquées d'utilisation du sol qui affectent nécessairement les conditions du régime hydrologique de ce secteur, notamment par une augmentation du ruissellement de surface. Les surfaces «minéralisées» des milieux urbains constituent en quelque sorte des «zones imperméables » aux précipitations et favorisent une augmentation du ruissellement superficiel (Konrad, 2003 ; Hollis, 1975), ce qui a pour effet d'alimenter plus rapidement le niveau des rivières et, conséquemment, d'augmenter les risques d'inondation.

\section{EXPANSION DES SURFACES URBANISÉES DES VILLES DE L'ESTRIE}

Pour illustrer les principales modifications anthropiques survenues au cours des dernières décennies, nous avons réalisé une cartographie détaillée des différentes villes étudiées, montrant l'expansion des surfaces urbanisées en bordure de la rivière Saint-François. Cette cartographie s'est faite à partir des photographies aériennes disponibles et ayant des échelles comparables. Les années retenues sont 1945, 1966, 1979 et 2000. Ces séries de photographies ont été rectifiées afin d'obtenir une plus grande précision des superficies calculées à partir des polygones ${ }^{3}$. Le tableau 5 fournit par ailleurs les données obtenues pour chacune des municipalités concernant l'augmentation de ces surfaces urbaines pour la même période (1945-2000), alors que le tableau 6 représente la répartition des surfaces

\footnotetext{
3 Les logiciels Geomatica (PCI) et ArcMap (ArcGis) ont été utilisés pour le travail de rectification et de cartographie des photographies aériennes.
} 
Tableau 4

Évolution de population des municipalités riveraines en l'Estrie ( 190 I-200 I)

\begin{tabular}{|c|c|c|c|c|}
\hline Inondation majeure & 1901 & |94I & $|98|$ & 2001 \\
\hline Bromptonville & - & 1672 & 3035 & 5658 \\
\hline Lennoxville & 1120 & 2150 & 3992 & 5004 \\
\hline Richmond & 2057 & 3082 & 3568 & 3540 \\
\hline Sherbrooke & 11765 & 35965 & 74075 & 77066 \\
\hline Windsor & 2149 & 3368 & 5233 & 5459 \\
\hline
\end{tabular}

Sources : Recensement Canada (190I, 194I) et Institut de la statistique du Québec (ISQ), 2008.

Tableau 5

Évolution des surfaces urbanisées entre la période de 1945 à 2000

\begin{tabular}{|c|c|c|c|c|}
\hline Villes & $\begin{array}{l}1945 \\
\left(m^{2}\right)\end{array}$ & $\begin{array}{l}1966 \\
\left(\mathrm{~m}^{2}\right)\end{array}$ & $\begin{array}{l}1979 \\
\left(m^{2}\right)\end{array}$ & $\begin{array}{l}2000 \\
\left(\mathrm{~m}^{2}\right)\end{array}$ \\
\hline Bromptonville & $\begin{array}{l}453224 \\
(3,96 \%)\end{array}$ & $\begin{array}{l}I|1| 582 \\
(4,56 \%)\end{array}$ & $\begin{array}{c}340 \text { I85 } \\
(4,38 \%)\end{array}$ & $\begin{array}{c}3735213 \\
(6,01 \%)\end{array}$ \\
\hline Lennoxville & $\begin{array}{l}885123 \\
(7,73 \%)\end{array}$ & $\begin{array}{c}2555082 \\
(10,48 \%)\end{array}$ & $\begin{array}{c}2270824 \\
(7,43 \%)\end{array}$ & $\begin{array}{c}3147004 \\
(5,06 \%)\end{array}$ \\
\hline Richmond & $\begin{array}{l}\text { I } 104604 \\
(9,65 \%)\end{array}$ & $\begin{array}{c}1986525 \\
(8,15 \%)\end{array}$ & $\begin{array}{c}2059914 \\
(6,74 \%)\end{array}$ & $\begin{array}{c}3646242 \\
(5,87 \%)\end{array}$ \\
\hline Sherbrooke & $\begin{array}{c}7896428 \\
(68,99 \%)\end{array}$ & $\begin{array}{c}16629347 \\
(68,22 \%)\end{array}$ & $\begin{array}{c}22496465 \\
(73,58 \%)\end{array}$ & $\begin{array}{c}46575608 \\
(74,95 \%)\end{array}$ \\
\hline Windsor & $\begin{array}{l}\text { I } 106432 \\
(9,67 \%)\end{array}$ & $\begin{array}{c}2094 I 25 \\
(8,59 \%)\end{array}$ & $\begin{array}{c}2406437 \\
(7,87 \%)\end{array}$ & $\begin{array}{c}5040313 \\
(8,11 \%)\end{array}$ \\
\hline Total & II 4458 II & 24376661 & 30573825 & 62144380 \\
\hline
\end{tabular}

Tableau 6

Répartition des surfaces urbaines, agricoles et boisées en bordure riveraine (tronçon Shebrooke-Richmond)

\begin{tabular}{|c|c|c|c|c|c|c|c|}
\hline \multirow[t]{2}{*}{ Rivière } & \multirow[t]{2}{*}{ Tronçon } & \multicolumn{3}{|c|}{$\begin{array}{c}\text { Rive droite } \\
\left(\mathrm{m}^{2}\right)\end{array}$} & \multicolumn{3}{|c|}{$\begin{array}{l}\text { Rive gauche } \\
\left(\mathrm{m}^{2}\right)\end{array}$} \\
\hline & & Urbain & Agricole & Boisé & Urbain & Agricole & Boisé \\
\hline $\begin{array}{l}\text { Saint- } \\
\text { François }\end{array}$ & $\begin{array}{l}\text { Sherbrooke/ } \\
\text { Bromptonville }\end{array}$ & 6396 & 6067 & 5411 & 8006 & 3593 & 5837 \\
\hline $\begin{array}{l}\text { Saint- } \\
\text { François }\end{array}$ & $\begin{array}{l}\text { Bromptonville/ } \\
\text { Windsor }\end{array}$ & 2599 & 5768 & 4643 & 3799 & 1336 & 7848 \\
\hline $\begin{array}{l}\text { Saint- } \\
\text { François }\end{array}$ & $\begin{array}{l}\text { Windsor/ } \\
\text { Richmond }\end{array}$ & 2779 & 8243 & 5491 & 4722 & 4080 & 8672 \\
\hline Total & & 11774 & 20078 & 15545 & 16527 & 9009 & 22357 \\
\hline
\end{tabular}

Compilation par les auteurs à partir des orthophotos de l'année 2000 (MRNF). 
urbaines, agricoles et boisées le long des rives de la Saint-François entre les villes de Sherbrooke et Richmond, réalisée à partir des orthophotos de l'année 2000 (MRNF, 2000) et des feuillets numériques (MRN, 2000). La bande riveraine analysée couvre une distance de 200 mètres de part et d'autre de la rivière. De manière générale, le trait dominant des modifications survenues au cours de ces années se manifeste essentiellement par l'implantation de nouvelles zones résidentielles et commerciales, et le développement de nouvelles infrastructures routières et de zones industrielles. Sur les différentes cartes obtenues (Figures 12 à 15), on peut facilement voir que le développement urbain s'accompagne d'une diminution au niveau de l'occupation agricole et des terres boisées. Par contre, on constate à certains endroits des gains en surfaces boisées au détriment des terres agricoles ou une densification des boisés existants, notamment le long de la rivière SaintFrançois.

La figure 12 présente l'évolution des surfaces urbanisées de la Ville de Sherbrooke entre 1945 et 2000, incluant la municipalité de Lennoxville, au sud. Celle-ci fait partie désormais de la grande agglomération de Sherbrooke, depuis les fusions municipales de 200I. On peut noter que l'étalement de la Ville de Sherbrooke s'est fait suivant deux axes principaux, soit l'axe vers le sud, suivant le couloir de la rivière Saint-François et la rivière Massawippi, et l'axe vers l'est et l'ouest, caractérisé soit par un développement résidentiel (axe/est), soit par la combinaison des activités industrielles ou manufacturières, accompagnée par le développement résidentiel (axe/ouest). On peut noter également que les surfaces boisées ont quelque peu diminué avec le temps, en particulier dans les zones soumises à une plus forte urbanisation près des quartiers centraux. Cet étalement urbain s'est fait au détriment des terres agricoles laissées en friche, ou encore de terrains boisés le long des rives. Par exemple, les massifs boisés localisés en bordure de la rivière Saint-François dans la portion nord de la municipalité de Sherbrooke ont été progressivement entamés pour faire place au développement résidentiel. On note une augmentation de la superficie urbaine de 7896428 à $16629347 \mathrm{~m}^{2}$ entre 1945 et 1966, alors qu'entre les années 1979 et 2000, elle passe à $46575608 \mathrm{~m}^{2}$ (Tableau 5). En incluant Lennoxville, on obtient une valeur de $49722612 \mathrm{~m}^{2}$ pour la période entre 1979 et 2000 , soit une hausse marquée des zones urbaines pour l'ensemble de cette agglomération ShebrookeLennoxville depuis 1945.
La municipalité de Bromptonville, située elle aussi en bordure de la rivière Saint-François, soit à une dizaine de kilomètres au nord-ouest de Sherbrooke, a connu une évolution plus lente mais constante de sa zone urbaine depuis les années 1945. Son extension la plus marquée survient surtout entre les années 1979 et 2000 (Figure 13). Entre 1945 et 1966, sa superficie urbaine est passée de 453224 à I ||| $582 \mathrm{~m}^{2}$, alors qu'entre les années 1979 et 2000 , elle augmente à $3735213 \mathrm{~m}^{2}$ (Tableau 5). Cette expansion urbaine de Bromptonville s'explique en grande partie par l'essor économique qu'a connu la Ville de Sherbrooke au cours des vingt dernières années (Gauthier, 200I), créant ainsi un pôle d'attraction économique important pour les municipalités environnantes, dont Bromptonville fait partie. Cette influence se manifeste notamment par le développement de nouveaux quartiers résidentiels localisés près des axes routiers, qui facilitent le transit entre Bromptonville et Sherbrooke. On note deux importants développements résidentiels, l'un dans l'axe sud-ouest du centre de Bromptonville et l'autre à quelques kilomètres au sud de la municipalité. En examinant le tableau 6, on note par ailleurs que les surfaces urbanisées le long des rives, entre Sherbrooke et Bromptonville, occupent des superficies plus importantes que les deux autres tronçons entre Bromptonville, Windsor et Richmond, en particulier sur la rive gauche, qui compte près du double de surfaces urbanisées. Le tronçon entre Bromptonville et Windsor indique aussi une portion non négligeable de surfaces urbanisées, notamment sur la rive droite, à la hauteur de la Ville de Windsor, qui forme toujours le centre urbain le plus important entre Bromptonville et Richmond, bien que cette ville souffre d'un déclin économique depuis les 30 à 40 dernières années (Proulx, 2002), ce qui se traduit d'ailleurs par une stagnation de sa population en général (Tableau 4). Enfin, le tronçon entre Windsor et Richmond s'apparente de beaucoup au secteur entre Bromptonville et Windsor, avec une plus faible représentation des surfaces urbanisées et une plus forte étendue des surfaces agricoles et boisées (Tableau 6). Sur la rive gauche, les terres boisées occupent une assez grande superficie, soit $8672 \mathrm{~m}^{2}$, ce qui dépasse quelque peu les valeurs obtenues dans le tronçon entre Sherbrooke et Bromptonville.

Enfin, pour les municipalités de Windsor et Richmond, on observe la même tendance que pour les autres municipalités à l'étude, soit une extension des surfaces urbanisées, surtout après les années 1966. Pour Windsor (Figure 14), elle a aussi profité, tout comme Bromptonville, de l'influence de la Ville de Sherbrooke dans son développement urbain, mais de 


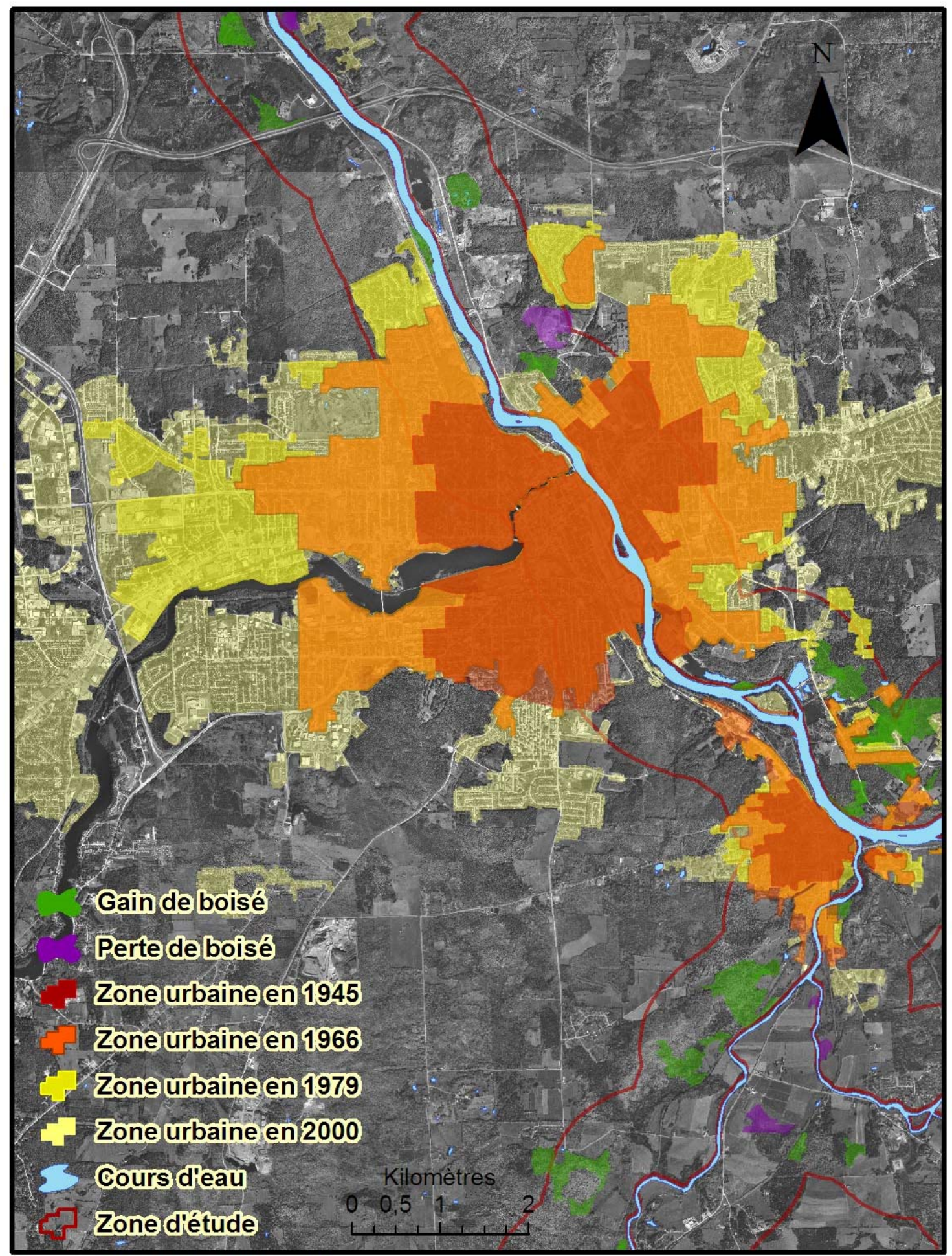

Sources: BNDT (2000), MRNF (2000).

Réalisation: Marlies Hähni (UQTR).

Fig. 12 - Évolution des surfaces urbanisées et boisées à Sherbrooke-Lennoxville (1945-2000) 


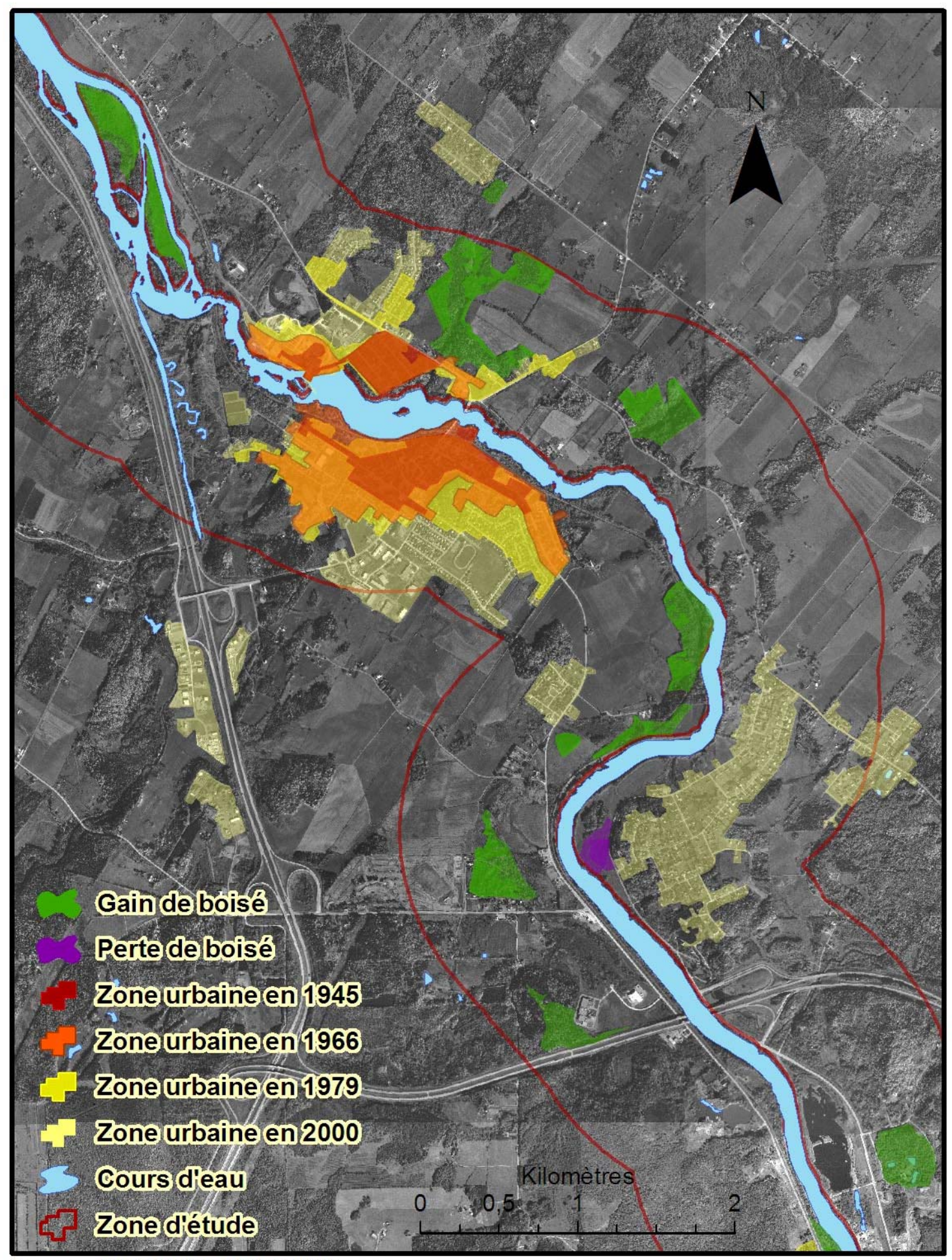

Sources: BNDT (2000), MRNF (2000).

Réalisation: Marlies Hähni (UQTR).

Fig. 13 - Évolution des surfaces urbanisées et boisées à Bromptonville (1945-2000) 


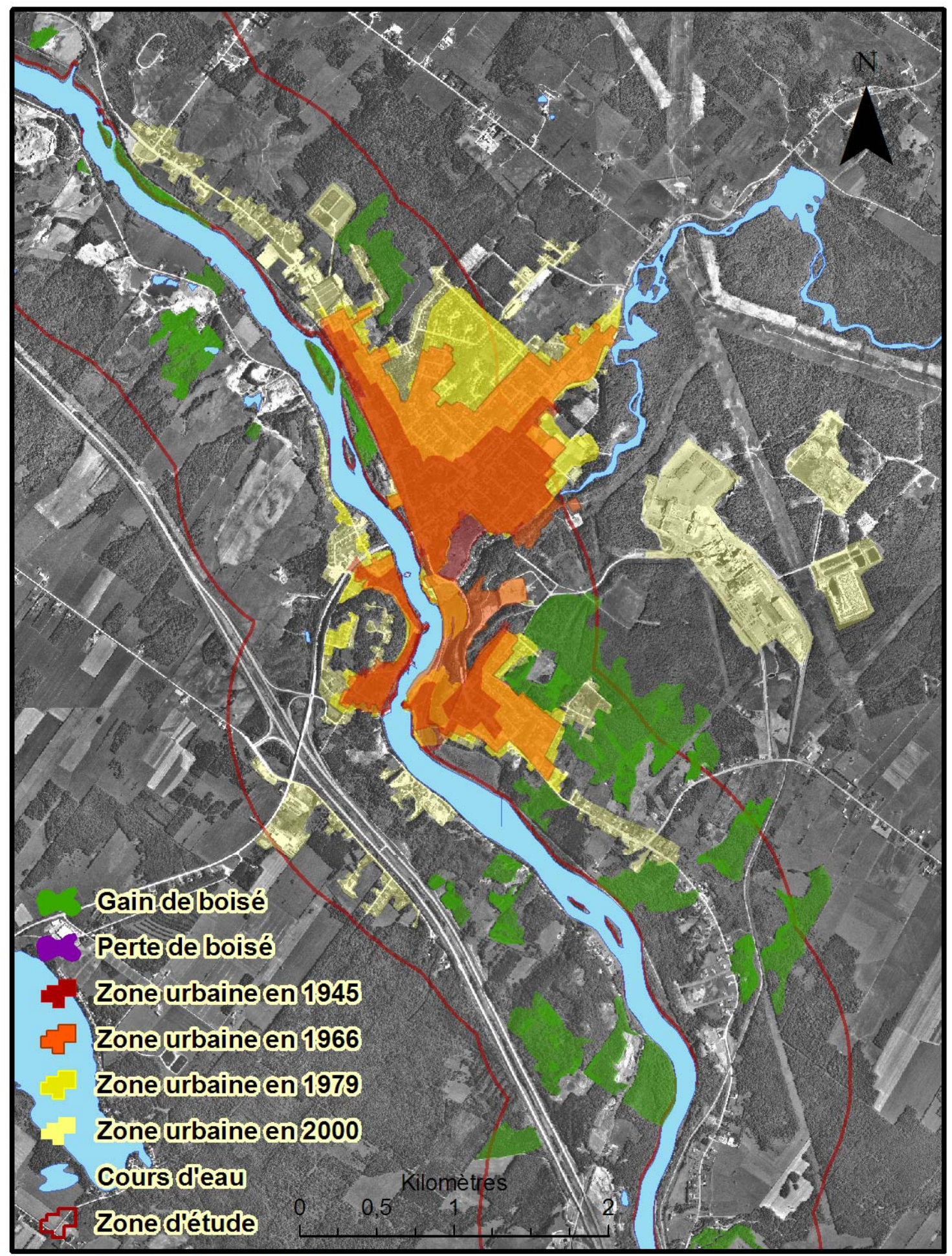

Sources: BNDT (2000), MRNF (2000).

Réalisation: Marlies Hähni (UQTR).

Fig. 14 - Évolution des surfaces urbanisées et boisées à Windsor (1945-2000) 


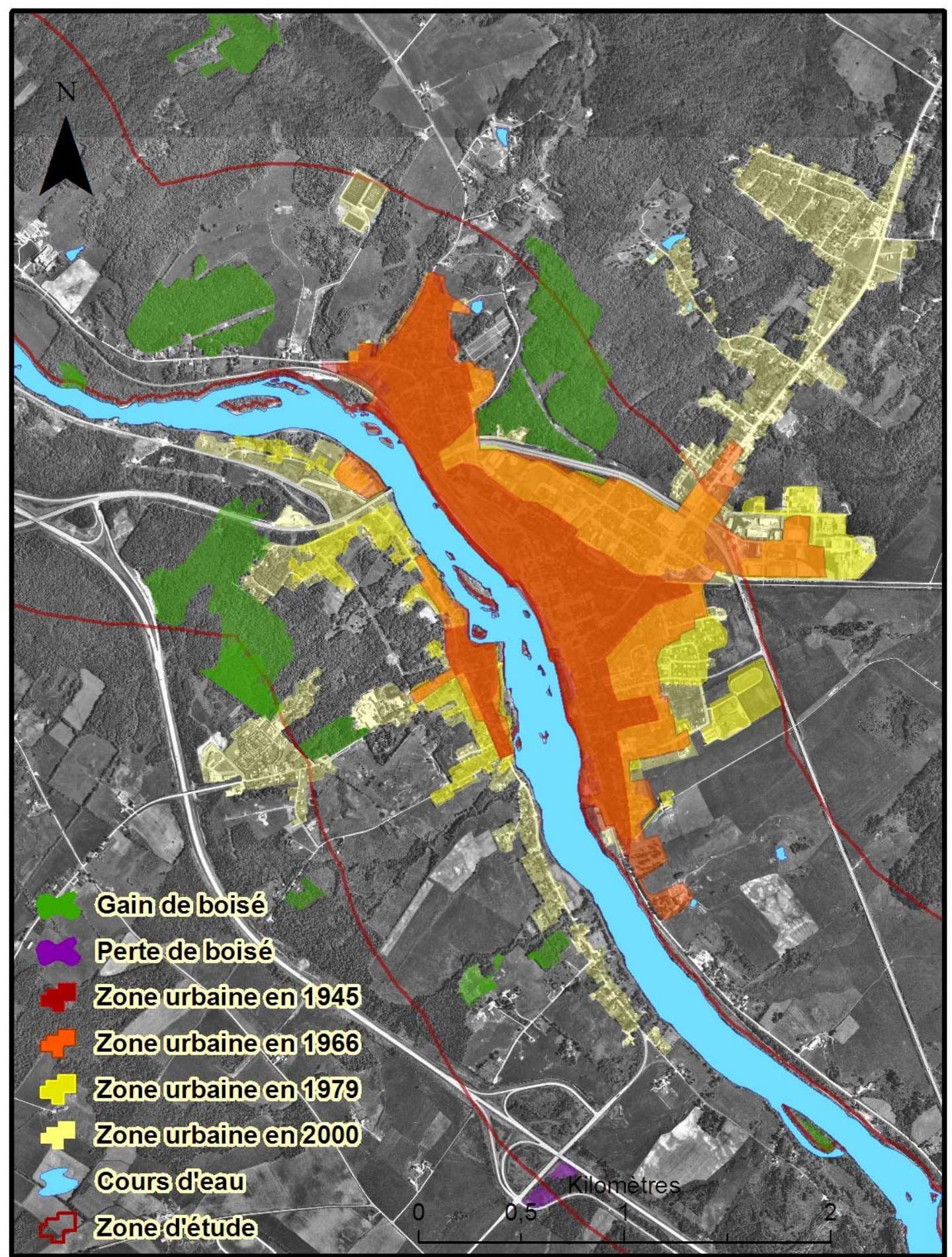

Sources: BNDT (2000), MRNF (2000). Réalisation: Marlies Hähni (UQTR).

Fig. I 5 - Évolution des surfaces urbanisées et boisées à Richmond (1945-2000) 
façon moindre, puisqu'elle est plus éloignée. Sa croissance est plus manifeste lors de la période de 1979 à 2000, passant de $2406437 \mathrm{~m}^{2}$ à $5040313 \mathrm{~m}^{2}$, soit une augmentation de $2633876 \mathrm{~m}^{2}$ en terme de nouvelles surfaces urbanisées. Cet étalement urbain s'est fait le long de la rivière Saint-François ainsi qu'en périphérie du noyau urbain, en direction d'un axe nord-est. Ce développement vers le nord-est s'explique par la relocalisation, en 1988, de l'usine Domtar inc. qui constitue l'une des plus importantes papetières de la région. Pour ce qui est de Richmond, l'augmentation est plus manifeste lors de la période de 1979 à 2000. Cette extension urbaine se fait surtout sur la rive droite de la rivière Saint-François (Figure 15), au détriment encore une fois des terres agricoles. Cet étalement urbain a suivi principalement l'axe de la route 116 vers Danville, située au nord-est. Enfin, toutes ces modifications survenues au cours des dernières décennies montrent à quel point les zones riveraines sont progressivement occupées par le développement urbain et qu'elles subissent, malgré la mise en place des règlements et politiques gouvernementales concernant la protection des rives et des plaines inondables (1987), l'assaut constant du développement urbain. Cette occupation progressive des rives ne fait qu'augmenter les surfaces vulnérables aux risques d'inondation et ce sont dans bien des cas les populations riveraines qui en subissent les premiers inconvénients. Et même si les risques d'inondation sont souvent vus comme des «catastrophes naturelles » inévitables, les constructions le long des rives et même dans les plaines inondables semblent se poursuivre à grande échelle, notamment dans les agglomérations qui connaissent un développement économique important, qui s'accompagne d'une croissance de leur population. On peut donc sérieusement s'interroger sur l'efficacité réelle des politiques et règlements qui régissent actuellement la protection des rives et des plaines inondables, en particulier pour les municipalités qui connaissent une forte croissance et qui sont tentées d'occuper ces espaces riverains très souvent convoités.

\section{CONCLUSION}

Cette étude porte essentiellement sur l'historique des inondations des villes de l'Estrie au cours de la période de 1900 à 2000. Les conditions hydroclimatiques et les modifications anthropiques survenues sur ce territoire en lien avec la fréquence des inondations sont aussi examinées avec les événements d'inondation. Les villes les plus souvent touchées par les inondations sont Sherbrooke, Bromptonville, Richmond et Windsor. Toutes ces villes sont situées le long de la rivière Saint-François et sont affectées, à divers degrés, par de fréquents débordements qui s'expliquent notamment par la configuration du réseau hydrographique. Sherbrooke se situe d'ailleurs à la confluence de nombreux affluents dont les rivières Magog et Massawippi. Les crues printanières sont souvent la cause principale des inondations, bien qu'on note des inondations majeures qui se sont produites durant la saison estivale, notamment les inondations de juin 1942 et 1943, qui furent parmi les pires inondations enregistrées au cours de ce siècle. Selon l'ensemble des données recensées, on note une hausse des inondations entre les années 1970 et 1990. Cette hausse est attribuée à une augmentation de la pluviométrie, elle-même responsable de la hausse marquée des écoulements en rivière durant la même période. Bien que les conditions climatiques constituent un facteur explicatif de premier ordre dans la récurrence des inondations de cette région, on doit aussi tenir compte des facteurs anthropiques qui interviennent aussi sur l'ampleur et l'intensité du phénomène, même s'il est difficile de mesurer leur impact de façon précise. Ces facteurs anthropiques se traduisent par des modifications progressives des milieux riverains, notamment par le déboisement et l'artificialisation des rives et l'extension des aires urbanisées dans les plaines d'inondation. Cette urbanisation progressive ne peut que contribuer à l'aggravation des inondations, en particulier dans les zones déjà périodiquement affectées par ce phénomène. Par ailleurs, il semble que la politique gouvernementale actuelle sur la protection des rives, du littoral et des plaines inondables (MDDEP, 1987) ne joue pas pleinement son rôle de protection pour ces zones à risques.

\section{BIBLIOGRAPHIE}

Barriendos, M., M.C. Llasat, A. Barrera et T. Rigo (2003). « The study of flood events from documentary sources: methodological guidelines for historical source identification and flood characterization in the Iberian Peninsula ", In V.R. THORNDYCRAFT, G. BENITO, M. BARRIENDOS et M.C. LlasAT, (Ed.), Palaeofloods, Historical Data and Climatic variability. Applications in flood risk assessment, p. 87-92. Proceeding of the PHEFRA International Workshops, Barcelona, 16-19th October, 2002.

BASE NATIONALE DE DONNÉES TOPOGRAPHIQUES (BNDT) (2000). Données géospatialisées. Ressources naturelles Canada, échelle I: 50000 , feuillets $2 \mathrm{IE}$ et $3 \mathrm{IH}$.

Boucher, R.J., P.E. BourbonNaIS, L. CARTIER et A. LeCLerC (1966). Bassin de la rivière Saint-François: Inondation des 45 novembre 1927, Étude hydrologique, Département des ressources hydrauliques, rapport 6-9, Gouvernement du Québec, 84 p. 
Boucher, R.J., L. CARTIER et A. LeCLerC (1953). Bassin de la rivière Saint-François: Inondation des 15 juin 1942, Département des ressources hydrauliques, rapport 6-83, Gouvernement du Québec, I35 p.

Boucher, R.J., L. CARTIER et A. LeClerC (1952). Bassin de la rivière Saint-François: Inondation des/5-16 juin 1943, Département des ressources hydrauliques, rapport 6-82, Gouvernement du Québec, $90 \mathrm{p}$.

Cartier, L. et A. Leclerc (1965). Bassin de la rivière SaintFrançois: Inondation des 10-II septembre 1924, Étude hydrologique, Ministère des Richesses naturelles, Rapport no 6-8, Gouvernement du Québec, 8I pP + 2 annexes.

Centre d'eXPertise hydRIQUe du Québec (CEHQ) (2000). Ministère du Développement durable, de l'Environnement et des Parcs (MDDEP), Gouvernement du Québec, [En ligne], [http://www.mddep.gouv.qc.ca/eau/regions/regions05/05 -estrie.htm], (consulté en décembre 2007).

COMMISSION DES EAUX COURANTES DU QUÉBEC (CECQ) (1952). Régime des eaux courantes de Québec. Rapports annuels couvrant la période de 1912 à 1952, Gouvernement du Québec, Québec.

COMMISSION DES EAUX COURANTES DU QUÉBEC (CECQ) (1944). Étude préliminaire des inondations - Bassin de la rivière Saint-François (juin 1942-1943), Rapport soumis par Léonard Cartier, I juin, 1944 (70-4-50).

Douglas, E.M., R.M. Vogel et C.N. Kroll (2000). « Trends in floods and low flows in the United States: impact of spatial correlation », Journal of Hydrology, vol.240, p. 90 105.

ENVIRONNEMENT CANADA (200I). Normales Climatiques Canada (197/-2000), Service Météorologique, Ottawa, Canada, [En ligne], [http://www.climate.weatheroffice.ec.gc.ca/climate normals/index_e.html] (Consulté en septembre 2007).

ENVIRONNEMENT CANADA (2002). Données hydrologiques, HYDAT CD-R. version 2.02 .

ENVIRONNement CANADA (2004). Adjusted Historical Canadian Climate Data (AHCCD), Climate Research Branch, Meteoroligical Service, Canada, [En ligne], [http://www.cccma.bc.ec.gc.ca/ahccd/] (Consulté en mars 2007).

EVANS J. et S. SCHREIDER (2002). « Hydrological impacts of climate change on inflows to Perth Australia », Climatic Change, vol. 55, p. 36I-393.

Gagnon, R.M., D.M. Pollock et D.M Sparrow (1970). Conditions météorologiques critiques et crues exceptionnelles des rivières Chaudière et Saint-François, Gouvernement du Québec, ministère des Richesses naturelles, direction générale des eaux, Service de la météorologie.

GAUTHIER, P. (200I). Rapport du mandataire gouvernemental concernant la réorganisation municipale dans la $R M R$ de Sherbrooke, Sherbrooke.
HOANG, V.D. (1982). Analyses des crues des 17 et 18 avril sur le bassin de la rivière Saint-François, Ministère de l'Environnement, feuillet météorologique, volume 21 : 127-133.

HolLIS, G. (1975). « The effect of urbanization on floods of different recurrence intervals », Water Resources Research, vol. II, p. 43I-435.

INSTITUT DE LA STATISTIQUE DU QUÉBEC (ISQ) (2008). Recensement de la population 200I, Gouvernement du Québec, [En ligne], [http://www.stat.gouv.qc.ca/regions/recens200I_05/pop ulation05/occupter05.htm], (consulté en janvier 2008).

JONES, N.K. (1999). «A recent history of flooding in the Massawippi drainage basin », Journal of Eastern Townships Studies, vol. 13, p. 4I-57.

JONES, N.K. (2004). «An analysis of the Massawippi Floods of 1982 and 1994 », Revue canadienne des ressources hydriques, vol. 29, p. 73-84.

JONES, P.D. et M. HULME (1996). «Calculating regional climatic time series for temperature and precipitation : methods and illustrations $»$, International Journal of Climatology, vol.16, p. 36I-377.

KONRAD, C.P. (2003). Effects of urban Development on floods, U.S. Geological Survey, (Fact Sheet 076-03), [En ligne], [http://pubs.usgs.gov/fs/fs07603/pdf/fs07603.pdf], (Consulté en janvier 2008).

LAMB, P. (1982). Analyse des pluies et de la fonte des 17 et 18 avril 1982 sur le bassin de la rivière de la rivière SaintFrançois, Ministère de l'Environnement, feuillet météorologique, 2I, p. 33-137.

LLAMAS, J. et J.P. TRIBOULET (1979). « Hydrologie du bassin moyen du Saint-Laurent ॥, Revue Canadienne des Ressources en Eau, vol. 4, p. 30-55.

Ministère des Richesses NATURELles (MRN) (1966). « Bassin de la rivière Saint-François - Inondation des 4-5 novembre 1927 », Étude hydrologique, vol. II, annexes, Rapport, 602, no. 6-9, Province de Québec.

Ministère des ResSources NATURelles et de LA FAune (MRNF) (2000). Photocartothèque québécoise, orthophotos 2000, échelle I :40 000, feuillets 2IE et $2 \mathrm{IH}$, Gouvernement du Québec.

Ministère des Ressources naturelles (2000). Base de données topographique du Québec (BDTQ), I: 20000 , feuillets numériques $2 \mathrm{IE}$ et $2 \mathrm{IH}$, Gouvernement du Québec.

MINISTÈre dU DÉVELOPPEMENT DURABLE, DE L'ENVIRONNEMENT ET PARCS. (MDDEP) (1987). Politique de protection des rives, du littoral et des plaines inondables, Gouvernement du Québec.

MONIRUL, M., Q. MIRZA, Q., R.A. WARRICK et N.J. ERICKSEN (2003). "The implications of climate change on floods on the Ganges, Brahmaputra and Meghna Rivers in Bangladesh », Climatic Change, vol. 57, p. 287-318. 
MUZIK, I. (200I). «Sensitivity of hydrologic systems to climate change », Canadian Water Resource Journal, 26, p. 233-252.

PANAGOUliA D. et G. DIMOU (1997). « Sensitivity of flood events to global climate change », Journal of Hydrology, vol. I9I, p. 208-222.

PIELKE, R.A. Jr. et W. DOWNTON (2000). « Precipitation and damaging floods. Trends in the United States, 1932 97 », Journal of Climate, vol. I3, p. 3625-3637.

ProulX, M.-U. (2002). L'économie des territories au Québec. Aménagement-Gestion-Développement, Presses de I'Université du Québec, Collection Science Régionale, $364 \mathrm{p}$.

ReCensement du Canada (190I). Population. Caractéristiques générales. Répartition géographique. Bureau fédéral de la Statistique, 4ième recensement, vol. I, Ottawa.

ReCensement du Canada (194I). Population. Caractéristiques générales. Répartition géographique. Bureau fédéral de la Statistique, 8ième recensement, vol. I, Ottawa.
Reynard, N.S., C. Prudhomme et S.M. Crooks (200I). «The flood characteristics of large U.K. rivers. Potential effects of changing climate and land use ", Climatic Change, vol. 48, p. 343-359.

Saint-laurent, D., C. Couture et E. McNeil (200I). "Spatio-temporal analysis of floods of the SaintFrançois drainage basin, Québec, Canada », Environments, vol. 29, p. 74-90.

Siew-Yan-Yu, T.O., J. Rousselle, G. Jacques et V.T.V NGUYEN (1998). «Régionalisation du régime de précipitations dans la région des Bois-Francs et de l'Estrie par l'analyse en composantes principales », Canadian Journal of Civil Engineering, vol. 25, p. 1050-1058.

Shrubsole, D., R. KreutzWiser, B. MitChell, T. Dickinson et D. JoY (1993). "The history of flood damages in Ontario ॥, Canadian Water Resource Journal, vol. 18, p.I33-I43.

TUCKER, C. (2000). « Floods in Canada: An Emergency Preparedness Canada (EPC) Perspective », Environments, vol. 28, p. 75-87 


\section{ANNEXE}

Recensement des événements d'inondation dans le bassin de la Saint-François et débits moyens journaliers maximums enregistrés à la station $020 F 002$ (1925-2000)

\begin{tabular}{|c|c|c|c|c|c|c|c|c|c|}
\hline $\begin{array}{l}\text { Date des inondations } \\
\text { recensées }\end{array}$ & $\begin{array}{c}\text { Débit maximal } \\
\text { enregistré lors } \\
\text { de } \\
\text { l'inondation } \\
\left(\mathrm{m}^{3} / \mathrm{s}\right)\end{array}$ & $\begin{array}{c}\text { Débit moyen } \\
\text { journalier } \\
\text { maximum } \\
\left(\mathrm{m}^{3} / \mathrm{s}\right)\end{array}$ & $\begin{array}{c}\text { Date } \\
\text { d'occurren } \\
\text { ce du Q } \\
\text { Max }\end{array}$ & Rang & $\begin{array}{l}\text { Date des inondations } \\
\text { recensées }\end{array}$ & $\begin{array}{c}\text { Débit maximal } \\
\text { enregistré lors } \\
\text { de } \\
\text { l'inondation } \\
\left(\mathrm{m}^{3} / \mathrm{s}\right)\end{array}$ & $\begin{array}{c}\text { Débit moyen } \\
\text { journalier } \\
\text { maximum } \\
\left(\mathrm{m}^{3} / \mathrm{s}\right)\end{array}$ & $\begin{array}{c}\text { Date } \\
\text { d'occurren } \\
\text { ce du Q } \\
\text { Max }\end{array}$ & Rang \\
\hline I925-Sep-II & 742 & 852 & Nov-17 & 54 & 1970-Avr-18-20 & 1590 & 1590 & Avr-19 & $19 *$ \\
\hline 1926 & - & 1390 & Mai-04 & 32 & |97|-Août-28-30 & 773 & 1280 & Mai-05 & $36 * *$ \\
\hline 1927-Nov-03-05 & 2110 & 2110 & Nov-05 & 5 & 1972-Mai-05-09 & 1680 & 1680 & Mai-06 & 16 \\
\hline |928-Mar-|4-I5 & 163 & 2360 & Avr-07 & 3 & 1972-Juil-16-17 & 385 & “ & “ & “ \\
\hline 1928-Avr-07-09 & 2360 & “ & “ & “ & 1972-Août-08-10 & 691 & “ & “ & “ \\
\hline I928-Mai-26 & 827 & “ & “ & “ & 1973-Juil-02-04 & 915 & 1550 & Mar-18 & 20 \\
\hline 1928-Oct-19 & 1120 & “ & “ & “ & 1974-Jan-29-30 & 453 & 1710 & Mai-0I & 14 \\
\hline 1929-Avr-09-10 & 1520 & 1520 & Avr-10 & 23 & 1974-Mar-08-09 & 869 & “ & “ & “ \\
\hline 1930-Jan-08-09 & 654 & 1170 & Avr-08 & 41 & 1974-Avr-04-05 & 1670 & “ & “ & “ \\
\hline |931-Avr-25 & 145 & 943 & Avr-12 & 52 & 1974-Avr-30-Mai-02 & 1710 & “ & “ & “ \\
\hline 1932 & - & 1640 & Avr-II & 17 & 1975-Août-27-28 & 240 & 1550 & Avr-20 & $20^{*}$ \\
\hline 1933-Avr-16-19 & 1990 & 1990 & Avr-19 & 8 & 1976-Mar-26-Avr-03 & 1850 & 1850 & Avr-02 & 10 \\
\hline |934-Avr-I |-13 & 1500 & 1500 & Avr-13 & 25 & |976-Août-I |-18 & 1160 & “ & “ & “ \\
\hline |935-Jan-I0-II & 762 & 852 & Avr-18 & $54^{*}$ & 1976-Déc-03 et 23 & 107 et 127 & “ & “ & “ \\
\hline 1936-Mar-13-19 & 2420 & 2420 & Mar-19 & 2 & | 977-Mar-I5-I7 & 1330 & 1330 & Mar-16 & $34 *$ \\
\hline 1937 & - & 1010 & Avr-2I & 48 & 1978-Avr-13 & 1030 & 1330 & Avr-2I & $34 * *$ \\
\hline 1938-Mar-23-24 & 1480 & 1480 & Mar-24 & 26 & | 978-Avr-2 I-24 & 1330 & “ & “ & “ \\
\hline 1939-Avr-22-25 & 2040 & 2040 & Avr-23 & 7 & |979-Jan-0|-05 & 428 & 1500 & Mar-26 & $25^{* *}$ \\
\hline I940-Mai-0I-03 & 1400 & 1400 & Mai-04 & 31 & 1979-Mar-05-08 & 949 & “ & “ & “ \\
\hline 1941 & - & 1230 & Avr-16 & 37 & |979-Juin-0 | & 521 & “ & “ & “ \\
\hline I942-Mai-I5? & 377 & 1970 & Juin-16 & 9 & 1980-Mar-17 & 417 & 498 & Avr-14 & 57 \\
\hline 1942-Juin-14-16 & 1970 & “ & “ & “ & |98|-Fév-22-25 & 1390 & 1390 & Fév-22 & 33 \\
\hline 1943-Juin-15-17 & 1740 & 1740 & Juin-17 & 13 & 1982-Avr-17-20 & 2420 & 2420 & Avr-19 & $2^{*}$ \\
\hline 1944 & - & 929 & Avr-26 & 53 & 1983-Fév-17 & 124 & 1690 & Déc-15 & 15 \\
\hline 1945-Mar-20 & 1510 & 1510 & Mar-19 & $24 *$ & |983-Mar-|9-2| & 985 & “ & “ & “" \\
\hline 1946 & - & 974 & Avr-13 & 51 & 1983-Sep-I7 & 44 & “ & “ & “ \\
\hline 1947 & - & 1440 & Avr-13 & 28 & 1983-Déc-07-22 & 1690 & “ & “ & “ \\
\hline | 948-Mar-| 9-22 & 1230 & 1230 & Mar-2I & $37^{*}$ & 1984 & - & 1070 & Juin-0I & 45 \\
\hline I949-Mar-23-24 & - & 1800 & Mar-28 & II & 1985-Fév-22-26 & 751 & 991 & Mar-30 & 49 \\
\hline 1950 & - & 1140 & Avr-2I & 43 & |986-Mar-26-3| & 1460 & 1460 & Mar-3I & 27 \\
\hline $195 \mid$ & - & 1300 & Avr-0I & 35 & | 987-Avr-0 I & 1540 & 1540 & Avr-0I & $21^{*}$ \\
\hline 1952 & - & 1020 & Avr-II & 47 & 1988 & - & 975 & Avr-05 & 50 \\
\hline 1953 & - & 1140 & Mar-28 & $43^{*}$ & 1989-Mar-15-16 & $44 I$ & $1780 *$ & Mar-29 & 12 \\
\hline 1954-Avr-I7 & 892 & 1090 & Avr-09 & 44 & |989-Mar-28-3| & $1780 *$ & “* & “ & “ \\
\hline 1955 & - & 1510 & Avr-16 & 24 & 1990-Mar-18-20 & $1410 *$ & $1410 *$ & Mar-18 & 30 \\
\hline 1956 & - & 1060 & Avr-30 & 46 & $1990-O c t-19-25$ & 1130 & “ & “ & “ \\
\hline 1957 & - & 1280 & Déc-22 & 36 & |990-Déc-22-3| & 1210 & “ & “ & “ \\
\hline 1958 & - & 1190 & Avr-23 & 39 & 1991-Avr-09-10 & $1620 *$ & $1620^{*}$ & Avr-10 & 18 \\
\hline 1959 & - & 1170 & Avr-07 & $4 I^{*}$ & 1992-Mar-10-13 & 930 & 1530 & Avr-23 & $22 *$ \\
\hline 1960-Avr-I-20 & 1590 & 1590 & Avr-05 & 19 & 1992-Avr-23 & 1530 & “ & “ & “ \\
\hline | 96 |-Juil-21-22? & 103 & 1140 & Avr-25 & $43^{* *}$ & 1993 & - & 1430 & Avr-II & 29 \\
\hline 1962-Mar-30-Avr-02 & 994 & 1530 & Avr-09 & 22 & 1994-Avr-17-18 & 2140 & 2140 & Avr-17 & 4 \\
\hline 1963-Mar-27-29 & 595 & 1280 & Avr-22 & $36 *$ & 1995-Jan-16-18? & 1157 & 1157 & Jan-18 & 42 \\
\hline 1964-Déc-12-15 & 129 & 1500 & Avr-16 & $25^{*}$ & 1996-Jan-17-25 & 2719 & 2719 & Jan-20 & 1 \\
\hline I 964-Mar-05-06 & 612 & “ & “ & “ & 1996-Fév-26 & 835 & “ & “ & “ \\
\hline 1964-Avr-15-16 & 1500 & “ & “ & “ & I996-Août-09 & 231 & “ & “ & “ \\
\hline 1965 & - & 564 & Avr-I7 & 56 & 1997-Fév-16 & 116 & 1218 & Avr-08 & 38 \\
\hline 1966 & - & 1060 & Mar-26 & $46^{*}$ & 1998-Mar-29-Avr-04 & 2050 & 2050 & Avr-0I & 6 \\
\hline 1967-Avr-03-04 & 1330 & 1330 & Avr-03 & 34 & 1999-Jan-25 & 218 & 686 & Avr-09 & 55 \\
\hline 1968 & & 1090 & Mar-24 & $44^{*}$ & 2000 & - & 1180 & Mai-II & 40 \\
\hline 1969-Avr-II-13 & 1540 & 1540 & Avr-18 & 21 & & & & & \\
\hline
\end{tabular}

